Hartwig Kalverkämper *

\title{
Die Wiederentdeckung des \\ kommunikativen Körpers - \\ Rhetorik, Theatralik und \\ Interkulturelle Wirtschaftskommunikation 1
}

\section{Körper - Kunst - Kommunikation}

Prähistorische Zeichnungen von Menschen, wie wir sie auf Felswänden in Höhlen bewundern können, strahlen eine befremdliche Distanz und eine unzugängliche Isoliertheit aus; sie mögen als rituelle Figuren auch einen besonderen Status innehaben - als Priester, Magier, Medizinmann, Hordenführer -, wir wissen nichts von ihrer gesellschaftlichen Stellung, vermuten diese aber aufgrund der besonderen Bildwirkung. Die Interpretation des „Enthobenseins“ aus der Gemeinschaft, der „Verbesonderung“ durch Funktion ist nur deshalb naheliegend, weil wir zwingend davon ausgehen können, daß der Mensch der Frühzeit ein

1 Dieser Beitrag ist im Rahmen einer Gastprofessur Mitte Februar bis Mitte März 1999 am Tysk Institut der Erhvervssproglige Fakultet entstanden, zu der mich die Wirtschaftsuniversität Aarhus (Handelshфjskolen i Arhus, Danmark) eingeladen hatte. Insbesondere die Thematik ,Dialogizität', die für eine skandinavische Konferenz während der Gastprofessur gewählt worden war, hat die Wichtigkeit des Aspekts ,Körperkommunikation" gerade auch in fachlichen Handlungszusammenhängen deutlich gemacht, so daß sich im dortigen genius loci ein interdisziplinäres Projekt (Körpersemiotik, Verständlichkeitsforschung / adressatenspezifische Texte-Optimierung, Rhetorik, Linguistik, Wirtschaftswissenschaft, Anthropologie) zur Interkulturellen Wirtschaftskommunikation anbot.

Für die herzliche Aufnahme am Institut und die wissenschaftlich außerordentlich anregende Atmosphäre sowie für den persönlichen und freundschaftlichen Umgang bedanke ich mich vor allem bei meinen dänischen Kolleginnen und Kollegen Marianne Ditlevsen, Jan Engberg, Peter Kastberg, Lisbet Maidahl Molly, Helmut Molly und Martin Nielsen. Tak for tiden i Danmark!

* Hartwig Kalverkämper

Humboldt-Universität zu Berlin

Institut für Romanistik

Unter den Linden 6

D-10099 Berlin

Hermes, Journal of Linguistics no. 23 - 1999 
Herdenwesen mit Rangordnungen und sozialen Bezügen und Aufgabenverteilungen war; in ein solches System passen die Unnahbarkeit und Vereinzelung - erst recht als Motive oder spürbare Merkmale von Zeichnungen - schwerlich. Auch die Malerei, Wandreliefs und Skulpturen der Frühkulturen zeigen Gottheiten und Repräsentanten der Führungskaste in seltsam anmutender Entrückung. Ihnen fehlt - aus heutiger Sicht betrachtet - etwas „Menschliches“, künstlerisch umgesetzte Signale der Conditio humana.

Die Kunst folgt der Gesellschaft; dies mag für die zweite Hälfte des zwanzigsten Jahrhunderts zwar nicht mehr unbestritten gelten, aber ansonsten spiegelt die Kunstgeschichte von der griechischen Antike an dieses Bedingungsgefüge von Produktion und Rezeption der Kunst in den jeweils gelebten gesellschaftlichen Dispositionen und Konstellationen wider. Der Mensch als Sozialwesen dürfte daher in Attika mit dem Aufbruch zur Demokratie ins Bewußtsein getreten sein, und so folgen die künstlerischen Darstellungen mit einem neuen Stil. Die überlieferten Skulpturen wirken kommunikativ, dialogisch-partnerbezogen, im wahrsten Sinne: , ansprechend" und folglich dem Betrachter näher und zugehöriger; und dies setzt sich in die römische Zeit weiter fort, nicht zuletzt angeregt und unterstützt durch das Kopieren (imitatio) griechischer Originale. Der kunstgeschichtlich einschneidende Wechsel, um solche Signalkraft auch für Nachfahren wie uns erfahrbar werden zu lassen, liegt weniger oder eher gar nicht daran, daß wir eine Skulptur, eine Statue, eine Zeichnung, eine Figur eines Wandfreskos im Idealfall ad personam identifizieren können; vielmehr ist es die Aussagequalität ihrer Körperhaltung, die sie nunmehr ,sozial(er)“ wirken läßt, sie in eine Lebenssituation einpaßt und Reaktion vom Betrachter verlangt.

Wer den menschlichen Körper als Mitteilungsform an den Betrachter wählt und ihn folglich als Anreiz oder sogar Aufforderung einsetzt, darauf (meist: in erwartbarer Weise) zu reagieren (zum Beispiel - wie wohl in den prähistorischen Höhlen - durch Demut oder religiöses Erschaudern; oder - wie bei den Griechen - durch ästhetischen Genuß; oder - wie bei den Römern - durch Respekt vor Autoritäten), der weiß um die Ausstrahlung körpergebundener Verhaltensweisen auf die Mitmenschen. Das gilt für den prähistorischen Höhlenmaler wie für den griechischen Bildhauer oder römischen Freskomaler. Und dieses Wissen verdichtet sich im Laufe der Kunstgeschichte als Tradition wach- 
sender Menschenkenntnis, der Schärfung von Beobachtung, der Gedanken zum Verhältnis von Emotion, Geist und körperlichem Ausdruck im wirkungsvollen Kunstwerk.

Das Mittelalter hat dazu die ganze Spannweite damaliger Befindlichkeiten künstlerisch umgesetzt: auf der einen Seite die adlige Selbstinszenierung, die in ihrer extensiven Körperbezogenheit kodifiziert war und somit ein eigenes Ausdruckspotential zur Verfügung hatte - der Alltag war ja nicht oder höchst selten (zum Beispiel auf Kalenderbildern in Gebetsbüchern [Belles Heures]) Thema der Kunst -; und auf der anderen Seite die Rücknahme der Körperlichkeit, bis hin zu einer gelebten Körperfeindlichkeit - in der Askese -, wie es die christliche Spiritualität als Form der Selbstfindung und des Gottesstrebens verlangte.

Die Renaissance-Künstler, die auf das wachsende Selbstbewußtsein des Individuums in der Gesellschaft reagieren und die Individualität des Menschen, zum Beispiel in den immer beliebter werdenden Portraits, beachten, haben dann den Gesichtspunkt der Wirkung durch den kommunikativen Körper in besonderer Weise umgesetzt; ihre Maler reflektieren die so geschaffenen Beziehungen zwischen Bildinhalt (Thema), Inhaltsträger (Person und ihre Darstellungsweise) und Bildwirkung auf den Bildbetrachter. So bemühte sich Leonardo da Vinci (1452 - 1519) in seinen vielen Studien und in seinen Werken, mit einer situationsangemessenen Lebhaftigkeit der Gebärden eine intensiv erfaßbare Lebensnähe zu schaffen. Sein Anspruch war es, die Absichten des Geistes durch Gesten und Bewegungen des Körpers eindringlich zu vermitteln. Mit dieser Einstellung zeigt er eine wesentliche, weil ganzheitlich ausgerichtete Weiterentwicklung gegenüber den Künstlern nördlich der Alpen, die noch begrenzter die menschliche Emotion und Ausstrahlung nur durch die Physiognomie und die Sprache der Hände, die Gestik, veranschaulichten. Absichten des Geistes - das bedeutet in einer partnergerichteten Aktion zugleich: Wirkung des Ausdrucksträgers, hier des Körpers; und folglich: Einwirkung auf die Reaktion des Gegenüber. Mit dem Körperausdruck beeindrucken und dadurch beeinflussen ist insbesondere in der Zeit der Gegenreformation ein didaktisch motiviertes Anliegen der Kunst des Humanismus: Gefühl und Glauben sind das Ziel, die Bewegung der Seele ist der Anspruch. Die bildende Kunst steigert dies im Barock zu Emphase, Affekt und Pathos. Sprachlich in der neuen Gattung ,Predigt` vereint, finden sich hier im Humanismus 
alte Wirkungsgesetze des aptum aus der antiken Rhetorik wieder, als Stillehren der schriftsprachlichen Texte, als Wirkungstheorie der mündlichen Rede wie auch der Musik (affektives Singen, eine Mischform von Gesang und Sprechen) und als Kunstauffassung der bildenden Künste und der Architektur.

Für die Gemeinschaft der artes bleibt somit die alte Rhetorik jener Zentralbereich, der am offenbarsten die enge Beziehung zwischen

- Redner (virtus dispositionis als iudicium des Redners zur gedanklichen [res] und sprachlichen [verba] Auswahl und Anordnung [dispositio, griech. oikonomiva oikonomía] der Teile seiner Rede),

- Sachanliegen (utilitas causae),

- Darstellungsweise (virtus elocutionis) und

- Publikum bzw. öffentlicher Reaktion (opinio, griech. dovxa dóxa) knüpft und auch analysiert und regelgeleitet lehrt.

Das Affektive und Emotionale als Mittel der Einwirkung, wie es im Begriff der persuasio (griech. peiv ${ }_{\mathrm{w}}$ peitho) als Mittel der Beeinflussung - neben der intellektuellen Überzeugung! - beschlossen ist, bot hier die Möglichkeit, eine Tradition der Disziplinen, Mentalitäten und Künste anzuschließen.

\section{Körperwirkung als Komponentenfunktion: ein integratives Kommunikationsmodell}

Die Wirkung des Körperlichen - was etwas anderes, nämlich bewußt Kommunikatives, ist als die Wirkung des Körpers - war also innerhalb der alten Künste nicht allein den Ausübenden der Bildenden Kunst bewußt. Zwar war und ist sie die Kunst, die durch die Beharrung des Augenblicks und durch die Bewahrung des Geschaffenen eine intensive Betrachtung ermöglicht und somit auch ein langes Studium in einer Tradition des Gelingens und Mißlingens sowie eines gewissen Fortschritts (Perfektionierung) ermöglicht; aber eine unmittelbare Wirkung, die sich als Einwirkung auf die Lebenspraxis und auf ein Steuern der Emotionen und Handlungen des Publikums zeigt, erreicht ihre Schwester, die Redekunst.

Sie legt, weil sie sich naturgemäß als mündliche und situativ sowie thematisch gebundene Rede verflüchtigt und nur in der Erinnerung wach bleibt, all ihre Wirkungsregister und Dynamik in den Augenblick, dabei in direkt beachteter Beziehung zur Reaktion des anwesenden Pu- 
blikums. Rasch ist die Zuhörerschaft auch als eine Zuschauerschaft erkannt worden. Und so hat sich auch hier ein Bewußtsein für die Wirkungspotenzen des mitredenden Körpers aufgebaut. Die antike Rhetorik widmet sich eigens dem Körpereinsatz, allerdings im Vergleich mit den systematischen Regelungen und subtilen Ratschlägen, wie sie für sprachliche Wirkungen eines gelungenen Redeeinsatzes vorgestellt werden, deutlich weniger und auch in den erkannten Kategorien erstaunlich gröber strukturiert.

Die beteiligten Komponenten sind damals wie heute abstrahierbar, nur scheint es weder für die theoretisch-wissenschaftliche Rhetorik des Aristoteles noch für die forensische Rhetorik des Cicero oder die wortgewaltige Predigtrhetorik der Reformation und Gegenreformation, auch nicht für die modernen, argumentationsbezogenen Rhetorik-Konzepte der Neuen Rhetorik von bevorzugtem Interesse gewesen zu sein, die körperbezogenen Ausdrucksformen und körperlichen Beeinflussungsmöglichkeiten näher zu untersuchen und zu strukturieren. Die Wirkungsmächtigkeit der aktiv im Redeprozeß eingesetzten Körperteile und darüber hinaus deren Ganzheitlichkeit als Haltung sowie die Eindruckskraft nonverbaler Wirkungsmittel wie Kleidung, Schmuck oder Geruch haben, schaut man sich überfliegend die lange Tradition der Rhetorik an, eigentlich nur in der pädagogischen Rhetorik (Ausbildung des vollkommenen Redners, Bildungsideal der Allgemeinbildung [griech. egkuvkloıpaideiva enkýklios paideía]) von Cicero (106-43 v.Chr.) in der republikanischen Zeit und von Quintilian (ca. 35 - ca. 100 n.Chr.) zur römischen Kaiserzeit näheres Interesse gefunden, wenn auch als facettenartige Erfahrungen einer geschulten Intuition. Etwa zweitausend Jahre später, in der Moderne des 20. Jahrhunderts, nimmt dann die Allgemeine Rhetorik, wie sie - als ,Tübinger Schule“ apostrophiert - mit Vertretern wie Walter Jens oder Joachim Dyck reduktionistische Konzepte überwinden will ${ }^{2}$, als kommunikationstheoretische Ausrichtung (Josef Kopperschmidt) und als praktische Rhetorik (rheto-

2 Dies ist nicht zu verwechseln mit der „Allgemeinen Rhetorik“, der Rhétorique générale, insbesondere aus den sechziger Jahren, wie sie von Vertretern aus dem sogen. „Lütticher Kreis“ vorgestellt worden ist (vgl. Dubois / Edeline / Klinkenberg / Minguet / Pire / Trinon 1970). Jenes Wiederaufleben der Rhetorik war zugleich verbunden mit neuartigen Interpretationen insbesondere der Figuren und Figurentheorie aus der alten elocutio-Lehre (mit zentraler Stellung der Metapher), die Brücken zur Linguistik, Narrativik, Kommunikationswissenschaft und besonders auch zur Semiotik schlugen (vgl. auch Fußn. 52). 
rica utens; Gert Ueding) gerade auch die äußeren wirkungsmächtigen und kommunikativen Formen der Rede gezielt in den Blick: massenmediale Kommunikation, Werbung, Wissenschaftssprache, Alltagskommunikation, sozialpsychologische Grundlagen von Sprechverhalten, soziokulturell bedingte Rezeptionsweisen, und dann natürlich auch die Wirksamkeit des Rhetorischen außerhalb der gesprochenen Sprache - in Bildern [,Rhetorik der Bilder'], Musik und selbstverständlich im Körperverhalten. Mit der Leiblichkeit von Kommunikation hat sich verstärkt die - sich ab den mittsechziger Jahren konturierende - Kommunikationspsychologie (Friedemann Schulz von Thun, Paul Watzlawik u.a.) befaßt, wenngleich nicht mehr in einem zentralen Selbstverständnis als ,Rhetorik'; für diese wäre eine solche Gewichtung als eine ,anthropologische Rhetorik“3 - eine Rhetorik, die sich der Körperkommunikation als menschlichem Ausdrucksmittel widmet - denkbar. Und in noch deutlicher geschärften Blick wird die Körpersprache dann von Autoren - Schauspieler, Pantomimen, Semiotiker, Ethologen - genommen, die sich in jüngerer Zeit, etwa ab den achtziger Jahren, dem Wirkungspotential bewußt gesteuerten Körpereinsatzes und unbewußter Körpersignale widmen (u.a. Samy Molcho, Desmond Morris, Irenäus Eibl-Eibesfeld).

Wenn nun, wie gesagt, die am kommunikativen Körpereinsatz beteiligten bzw. mit ihm unlösbar zusammenhängenden Komponenten isoliert werden können, sei dies in sechs Schritten (2.1. bis 2.6.) hier vorgeführt:

2.1. Mit den beiden anderen artes in Gemeinschaft des trivium - nämlich außer der rhetorica die grammatica und die dialectica - wird ein Grundstock gelegt und ausgebaut, der einen europäischen, besser noch: abendländischen Begriff von ,KULTUR` zu prägen beginnt; hier liegen die Quellen einer Mentalitätsgeschichte, aus der wir in der heutigen Zeit als konstitutive Qualität des ,Kultur'-Begriffs die Dynamizität angeben, und als prinzipielle Komponenten den homo sociologicus, das Gemeinschaftswesen, und den homo faber, das Wirkwesen (wie es sich im fachlichen, im arbeitsteiligen Handeln zeigt), beide vereint durch differenziertes kommunikatives Verhalten (homo loquens - der Mensch als kommunikatives Wesen).

3 Kalverkämper (1998b: 1362-1364). 
Die konstitutiven Züge eines (abendländischen), Kultur'-Begriffs sind dynamisch, prozessual ausgelegt und betreffen im Kommunikationsablauf, im dialogischen Prinzip, grundsätzlich den homo sociologicus und den homo faber (vgl. Kalverkämper 1995; 1998a; 1999b). Es ist somit kaum möglich, eine einfache Wesensdefinition für das komplexe Phänomen bzw. den vielfältig ausgelegten Begriff zu bieten; hier erscheint allein die Bestimmungsgemeinschaft einer definitio per proprietates angebracht. Ich schlage folgende Komponenten vor, die gemeinsam für einen ganzheitlichen ,Kultur'-Begriff stehen:

1. Auf den homo sociologicus richten sich folgende Aspekte:

a die identifikationsstiftende gleiche Sprache als gemeinsame Bindung;

a die kollektiv empfundenen Wertvorstellungen und verhaltenssteuernden Inhalte;

ø identitätsstabilisierende äußere Manifestationsweisen von Werten, Zielen, sozialen Erfahrungen;

a tradierte Formen des Feierns und des Gedenkens;

a Konsens bei der Gestaltung und Strukturierung des Alltags;

x als unabdingbar und sozial verbindlich angesehene Markierungen im Lebenslauf der einzelnen Mitglieder der Gesellschaft;

a konventionalisierte Formen des Umgangs miteinander und der sozialen Ordnung;

a gemeinsam akzeptierte und kaum in Frage gestellte Einschätzungsgrundlagen gegenüber Fremdem, Andersartigem, Neuem was sich insbesondere in Klischees und gefestigten Vorurteilen zeigt.

2. Den wirkenden Menschen betrifft die Kategorie des homo faber, der mit folgenden Komponenten den ,Kultur'-Begriff mitkonstituiert:

a kunstschaffender Ausdruckswille in Literatur und den Künsten und deren Selbstverständnis in der Auseinandersetzung zwischen traditionsbezogener Beharrung und zukunftsgerichteter Veränderung (Konstanz und Variation; Statik und Dynamik);

○ weitgehender Konsens bei der Gestaltung und Bewertung von Arbeitsprozessen (,Beruf‘, Zugehörigkeit zu einem Fach oder einer Disziplin, soziale Rolle durch Fachwissen, gesellschaftliche Reputation von Ausbildung und geschulten Kenntnissen, Integra- 
tion von Wissenschaft in gesellschaftliche Meinungsbildung und Akzeptanz, Stellenwert von Allgemeinwissen, Einschätzung des Wertes von Arbeit);

a forschender Gestaltungsdrang in den Wissenschaften und Technologien, was eng mit ,Fortschritt' verbunden ist, dabei aber auch einbezieht:

a Kontakte des Transfers, Kooperation und Wettbewerb; einschließlich und unverzichtbar die Bereitschaft und Fähigkeit zu einer philosophischen Begleitung, zu einer ethischen Rückbesinnung und Korrekturoffenheit, zu einer moralischen Reflexion, zu einer kritisch-sozialen Inspektion, zu einer Sensibilisierung für Grenzen des Machbaren und für eine prinzipielle Verantwortung vor der Zukunft.

2.2. Die beiden artes bringen zudem die MEDIALITÄTEN zusammen, nämlich die (originäre und somit primäre) Mündlichkeit (Rhetorik) und die Schriftlichkeit und ihre Varianten des Memorierens (Bildende Kunst). Auch hieran läßt sich eine Tradition anschließen, die in der systematisierenden Zuordnung heutiger Zeit mündet, indem Medialität und Semiotizität in Beziehung gebracht werden: das Korrelat der mündlichen Texte außerhalb der Sprache ist die Körpersprache; das Korrelat der schriftlichen Texte außerhalb der Sprache sind die Abbildungen (Zeichnungen, Grafiken, Diagramme, Photos, usw.).

SEMIOTISCHE DIMENSION




2.3. Auch das Verwobensein von Ausdruck - sprachlichem wie bildnerischem - wird von beiden Künsten in ihren jeweiligen Eigenarten vermittelt: die Bildende Kunst komponiert die Einzelteile zu einem aussagestarken Ganzen, dem Kunstwerk, und schafft so eine ganzheitlich wirkende Aussage (Schmerz, Glück, Erwartung, Huldigungsanspruch, Demut, usw.). Die Rhetorik ihrerseits ist äußerst sensibel bedacht auf die Wirkung ihres Ganzen, nämlich der Rede, und sieht mit Recht dies durch die spezifischen Funktionen der Teile und ihrer Abfolge und gegenseitigen Bezüge gegeben. Beide artes sind also genuin TEXT-gerichtet (lat. textum, das Gewebe"; texere ,[ver]weben').

2.4. Der dahinterstehende Aufforderungscharakter läßt sich kaum leugnen: Kunst braucht Öffentlichkeit, will Publikum, lebt von der Resonanz, verlangt Deutung, somit Partnerbezug, folglich Dialog. Das ist auch das Spektrum, das mit dem Brennpunkt ,Dialogizität‘ das Rhetorische umgreift, denn gerade hier wird das Miteinander-Sein konstitutiv: wo kein Publikum, da keine Rhetorik. Und da sich Publikum als Ausschnitt aus gesellschaftlicher Öffentlichkeit nur dort zusammen,,stellt“, wo Gemeinsamkeiten vorhanden sind, repräsentiert die genuin dialogische Qualität von Rhetorik auch Grundsätzliches des homo sociologicus, des homo loquens, des homo agens: die Kommunikation. Etymologisch bedeutet dieses Wort, das aus communis esse stammt, ,gemeinsam sein', eigentlich ,gemeinsame Mauern habend' oder, in gemeinsamen Mauern vereint seiend' (lat. cum und moenia, letzteres von munire , befestigen'). Es ist darin die Unausweichlichkeit der Zuwendung, der Bezug der Körper und Sinne beschlossen. Die „kommunikative Dyade“ (Weinrich 1993: 17f.) (oder - weniger angloamerikanisch, näher am Griechischen - ,Dyas') ist principium humanum. Sie ist ausgelegt als AKTION und Prozeß, begreift sich also dynamisch.

2.5. Um das Funktionieren der kommunikativen Dyade zu gewährleisten, stehen Mittel - Vermittler - zur Verfügung: die SPRACHE, wobei man sie auch in einem metaphorischen Sinn für die Kunst verstehen kann: Sprache der Kunst, künstlerische Sprache mit Ausdruck und Inhalt, wie man es von der ,Wortsprache“, die ja erst als „Sprache in Texten“ funktioniert (Weinrich 1976), her kennt. Vom kleinsten bedeutungstragenden Zeichen („Lexik“) über seine Zusammenbindung (,Syntax“) mittels dazu definierter Organisationsmittel (,Morpholo- 
gie“) aus einem systematischen Ordnungssystem (,Grammatik“) hin zu einem funktionalen Zusammenspiel, verwoben als Text, bezogen auf sortenspezifische Rahmenbestimmungen (,Textsorte“, „Gattung“, „Verhaltensmuster“), gebunden an die Lebenssituation, Umstände und Konstellationen (,Pragmatik“), eingebettet in soziokulturellen Kontext (,Kultur") - das ist das Format, mit dem das Kommunikationsmittel funktioniert. Die Rhetoren haben dies schon in der Antike erkannt und sich insbesondere dem sprachlichen Anteil gewidmet ${ }^{4}$, dabei aber - wie schon gesagt (s.o. 2.) - auch dem körperlichen Anteil zugewandt. ${ }^{5}$ Die begriffliche Formel des komplexen Formats, wie Sprache funktioniert nämlich als Sprache-in-Texten-in-(Kommunikations-)-Situation(en)in-Kultureinbettung ${ }^{6}$ - hat folglich analoge Geltung für die Bestimmung von Körpersprache. Kommunikation ist immer kultureingebettet, immer Manifestation von Kulturemen (also den konstitutiven Einheiten von ,Kultur'; s.o. 2.1.), die Identität verleihen, Werte vorgeben, Erwartungen entstehen lassen, Beurteilungen ausprägen, Verhaltensweisen steuern.

2.6. Die Dyade und das Kommunikationsmittel sind natürlich gebunden an ihren Träger, die konkrete PERSON, die aussagen will, die auf den anderen (ein-)wirken will, die Reaktion erwartet, Handlungen vollzieht und dabei Verhaltensweisen zeigt, die ihrerseits von der Kul-

4 Strukturelle Konstanten im rhetorischen System sind

- die Trennung nach ,in verbis singulis “ und ,in verbis coniunctis “ und

- die Beachtung von „compositio“ sowie

- die Berücksichtigung der Kategorie ,aptum “ (vgl. 3.2. und Fußn. 52)

(s. Lausberg ${ }^{10} 1990$; vgl. auch Kalverkämper 1983 und 1999a).

5 Man könnte aus heutiger Sicht analog zum oben dargestellten systematischen Aufbau von ,Sprache" auch das System von ,Körpersprache" mit seinen integrierten Komponenten jeweils zunehmender Komplexität beschreiben:

- Ein körpersprachliches Lexikon, systematisch angelegt,

- eine Syntax, organisiert als Grammatik,

- ein funktionales Zusammenspiel, verwoben als (Körper-)Text,

- bezogen auf (kommunikations-) sortenspezifizierende Rahmenbestimmungen,

- gebunden an die Pragmatik von Kommunikation,

- eingebettet in die Kultur

(vgl. Kalverkämper 1995: 143-145; 1997: 215-219; 1998b: 1346-1348).

6 Diese wissenschaftssystematische und methodologische Position habe ich insbesondere aus der Sicht der Fachkommunikationsforschung, die dafür besondere Evidenzen bietet, seit 1983 formuliert und in verschiedenen Arbeiten vertreten; summierend in Kalverkämper (1996: hier insbes. 155f.) oder (1998d: insbes. 42), sowie unter translationswissenschaftlichem Fokus in Kalverkämper (1999b). 
turgemeinschaft „geprüft“ werden, woraus sich dann wieder Schlüsse (z.B. Akzeptanz wegen Normkonformität; z.B. Befremden oder Ablehnen wegen Erwartungs- oder Normverstoß) ergeben, die wiederum für das Individuum und sein Kommunikationsverhalten wichtig sind.

2.7. Die Komponenten, die hier in Vereinzelung vorgestellt worden sind (2.1. bis 2.6.), stehen in einem prozessualen und hierarchischen Verhältnis zueinander. Man kann die Beziehungen in einem Inklusionsmodell (s. nächste Seite) fassen. Die Kategorien PERSON (2.6.), SPRACHE (2.5.), HANDLUNG (2.4.), TEXT (2.3.), MEDIUM (2.2.) und KULTUR (2.1.) sind hier als Eigenschaften der Kommunikation, mit Adjektiven, repräsentiert: die PERSONALE Voraussetzung (,wer“?) verlangt nach dem zentralen Platz in dem Modell; die LINGUALE Voraussetzung zum Kommunizieren (,womit“?) manifestiert sich als TEXTUELlE Ganzheit in einem AKTIONALEN Ablauf, die beide, die Sprache umschließend, gleichberechtigt miteinander verbunden sind (,wie“?); alles Kommunizieren verdeutlicht sich über Bedeutungsträger, ist also MEDIAL gebunden (,,mit welchen Mitteln“"?); es geschieht in KULTURspezifischen Rahmen, die die Kommunikationsweise (Medium) und die Sprachhandlungen und Vertextungen wie auch die Sprache bestimmen oder zumindest beeinflussen und auch die Personen selbst als Glieder der Gesellschaft, der Kulturgemeinschaft, prägen: nämlich durch Erwartungsvorgaben für die Produktion und Rezeption. 


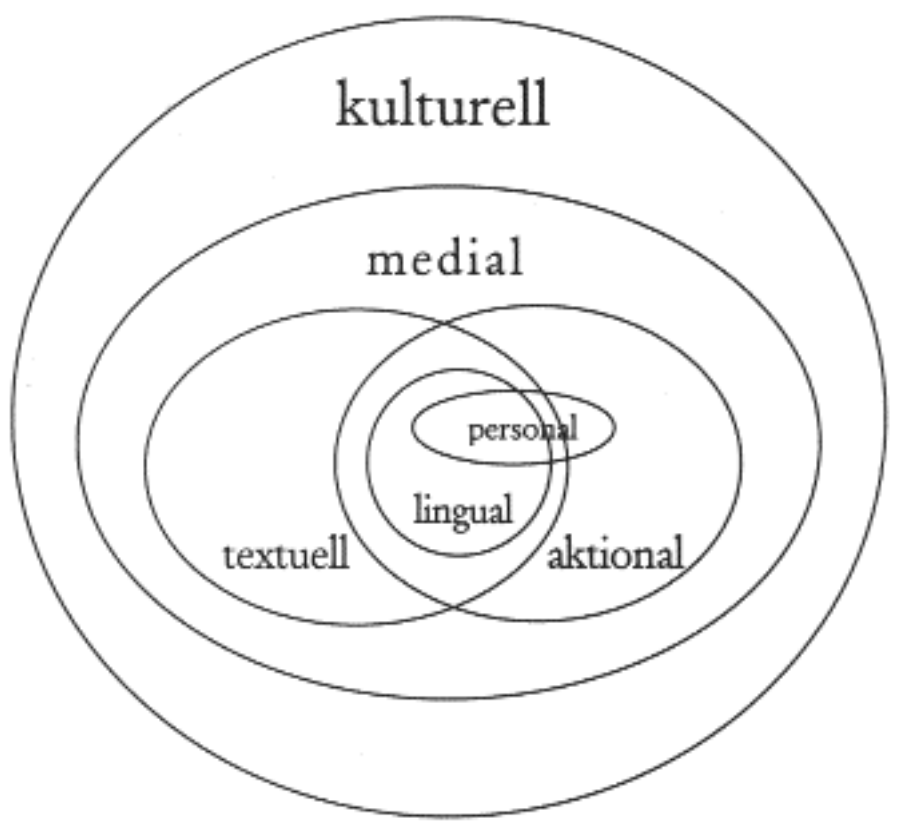

\section{Körperkommunikation als Kategorie der antiken Rhetorik(praxis)}

Das Modell liefert eine universale Beschreibung der hierarchischen Beziehungen, die zwischen den unabdingbaren Komponenten von Kommunikationsprozessen herrschen. ${ }^{7}$ Mit dieser Leistung bietet es dann auch eine spezifische Erfassung der Zusammenhänge, die für den Einsatz von Rhetorik gelten. ${ }^{8}$

Funktional gesehen verstand und versteht sich die Rhetorik zwar generell als ars, d.h. als eine ,von einem vernünftigen Wesen (Mensch)

7 Mein Vorschlag soll auch dazu verhelfen, das „übliche“ Kommunikationsmodell, dessen Trivialität (Sender, Empfänger, Text, Code oder Sprachsystem, seit den spätsiebziger Jahren noch Situation, ab den neunziger Jahren zudem Kultur; s. Kalverkämper 1999b: 66) nur durch verschiedene Grade der Feindifferenzierungen interessant gemacht wird, durch ein Integrationsmodell zu bereichern.

8 Es leistet auch einen spezifischen Erklärungswert bei translationswissenschaftlichen Überlegungen (vgl. Kalverkämper 1999b). 
planvoll ins Werk gesetzte Handlung“ (Lausberg 1960, §1)9, als eine ,Technik“ (griech. tevcnh téchne ${ }^{10}$ ) und somit als ,ein System aus der Erfahrung (griech. empeiriza empeiría) gewonnener, aber nachträglich logisch durchdachter, lehrhafter Regeln zur richtigen Durchführung einer auf Vollkommenheit zielenden, beliebig wiederholbaren Handlung, die nicht zum naturnotwendigen Geschehensablauf gehört und nicht dem Zufall überlassen werden soll“" (Lausberg 1960: §3).

Die Ansichten zum Wesen der Rhetorik in ihrem Selbst- oder auch Fremdverständnis wechselten im Laufe der europäischen Mentalitätsgeschichte. Curtius (1938: 135) zum Beispiel hat das Schillern des Begriffs ,Rhetorik' bereits für die antike Zeit aufgezeigt, woraus eigentlich zu schließen ist, nicht einfach von ,der Rhetorik“ ausgehen zu können, was im übrigen auch schon von Quintilian selbst diskutiert wird (II 14, 1-5), um sie dann (II 14, 5) als bene dicendi scientia festzulegen; und Kopperschmidt (1973: 13) erkennt auch für den heutigen Sprachgebrauch verschiedene Verwendungsweisen (,Redetheorie“ / ,Redelehre', ,Redekunst' und ,Redefähigkeit ') oder Spillner (1977: 94) unterscheidet vier Auffassungen (,Technik' / ,Anleitung zur Praxis', ,Theorie zur Produktion persuasiver Texte', ,Instrument für Textanalyse', ,Mittel für Erkenntnis- und Handlungsprozesse ').

3.1. Es ist auffallend, daß die Rhetorik in den Wesensbestimmungen so stark mit dem Verbalen identifiziert wird. Dabei haben Quintilian ${ }^{11}$ und, rund zweihundert Jahre vor ihm, schon Cicero ${ }^{12}$ darauf hingewiesen, daß sie gerade auch eine ars in agendo posita (tev cnh praktikh téchne praktike), eine „Aufführungskunst“13 mit einer Handlung (actio) und mit Handlungsbeteiligten (actor) ist, was ja die situative Gebundenheit und die direkt sinnenfällige Öffentlichkeitswirkung - also den pragmatischen Wesenszug - herausstreicht. In der Tat kommen hierin die Komponenten des Modells zum Tragen: in agendo, das ist der Aktant als das Medium, handelnd in der Gemeinschaft, auf

9 ,Geregelte Kunst', ,körperliche oder geistige Fertigkeit', ,Handwerk', ,Wissenschaft".

10 ,Geschicklichkeit‘, ,Kunstfertigkeit‘, ,Handwerk‘; , geistige Gewandtheit‘.

11 Quintilian XI 3, 1-184.

12 Cicero, De oratore III 56, 213 bis III 61, 227; Cicero, Orator 17, 54 bis 18, 60.

13 Lausberg (1960: $§ 10,2)$. 
der agorga agorá, dem forum, dem Markt, beim Gericht, im gumnajsion gymnásion, in der akadhmiza akademía. Dort wird die Rede verwirklicht (,wirken'!), durch Sprechen und Körpereinsatz. Cicero (de oratore III 56, 513) und Quintilian (XI 3, 1) trennen hier folgerichtig nach pronuntiatio (,Vortrag', ,gesprochene Rede') und actio (,Auftreten', griech. (ópojkrisi ı hypókrisis), um das Zusammenspiel von vox atque motus (,Stimme und Bewegung '; Quintilian XI $3,1) \mathrm{zu}$ erfassen: pronuntiatio est ex rerum et verborum dignitate vocis et corporis moderatio (Cicero, De inventione I 7, 914). Das Kapitel XI 3 (,pronuntiatio“) der Institutio oratoria berücksichtigt die Wirkung auf die Partner, die Zuhörerschaft, die Öffentlichkeit, die Gesellschaft, indem es auch die Affektenlehre (affectus) mit einbezieht. So bietet es sich als eine kommunikationspraktische Abhandlung an, die

- kunstvolles Sprechen,

- Gattungsspezifik,

- Körpereinsatz und

- Wirkungsweisen auf den Gegenüber

als funktional miteinander verwoben beschreibt.

Die Antike hat dieses Zusammenspiel immerhin als so konstitutiv angesehen, daß sie auch für ihre literarischen Gattungen, die der pragmatischen Rhetorik als artes in agendo positae zur Seite stehen, die körperlich ausdrucksgebundene Vortragsweise beachten: beim Vortrag der Epen (nJaywdein rhapsodein), der Lyrik (diavdein diádein) und des Dramas (hJ uJpokritikhv he hypokritiké) ${ }^{15}$.

Ein ganzheitliches Lehrprogramm hat sich allerdings für die nichtsprachliche Komponente des Vortrags daraus nicht entwickelt; vielmehr blieben das Zusammenspiel und die Gesamtwirkung im Blick: „die Stimme, die schöne Haltung, die sich im Ausdruck dem anpaßt, was die Stelle gerade verlangt, die Art des Vortrags, die wohl das Aller-

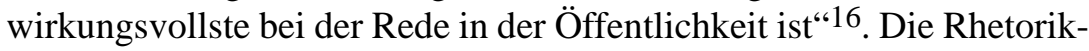
lehrer der Antike haben zwar das Phänomen als solches und in seiner Bedeutung und seiner Wirkungsmächtigkeit erkannt, doch haben sie

14 ,Ein Vortrag ist das wohlabgewogene Maß von Stimme und Körper aus der eindrucksvollen Fülle möglicher Inhalte und Sprachmittel'.

15 Aristoteles, Poetik 26, 6. - Vgl. Lausberg (1960: §1091).

16 vox, actio decora, commodata, ut quisque locus postulabit, pronuntiandi vel potentissima in dicendo ratio (Quintilian X 1, 16f.). 
den Körper mit seinen kommunikativen Verhaltensweisen nicht einer vergleichbaren detaillierten Systematisierung mit Kriterien, Regeln, Wertungen und Wirkungsprognosen unterzogen und für die Lehre aufbereitet wie die öffentlich gesprochene, später dann (als, literarische Rhetorik') die geschriebene Sprache. Eher lesen sich die kapitelgebundenen, somit eigens herausgestellten Ausführungen bei Cicero oder Quintilian als eine Sammlung von Anweisungsrezepten aus langer Erfahrung und somit aus geschulter Intuition, als eine praktische Psychologie - sogar als eine Psychagogik - zur partnerbezogenen Körperwirkung, zur publikumssteuernden Selbstdarstellung und zur einflußnehmenden Präsentation der Sachinhalte. ${ }^{17}$

Der Begriff sermo corporis, selbst eine Metapher, von Cicero in De oratore (III 222) eingebracht, meint somit noch deutlich angemessener als ,Körpersprache“ eigentlich das ,Gespräch“ mit Hilfe des Körpers, worin der dialogische (,dyadische') Charakter des communis esse, der gemeinsamen Kommunikationssituation, anklingt. Damit stellen die beiden bedeutendsten Redner - Redetechniker und Redetheoretiker der Antike die Verbalsprache und die Körpersprache auf eine gleiche Gewichtigkeitsebene; Cicero (De oratore III 223) sieht im körperlichen Ausdrucksverhalten sogar den Vorteil gegenüber der Einzelsprache, daß alle Menschen von den Gesten und Regungen gleichermaßen berührt werden ${ }^{18}$; und Quintilian faßt dies noch prägnanter, indem er die Körpersprache erhöht zu einem Ausdrucksrepertoire, das übereinzelsprachlich, universal gilt und verstanden werde, als die ,gemeinsame Sprache der Menschheit“ (omnium hominum communis sermo) ${ }^{19}$. Daß

$\overline{17}$ atque in eis omnibus, quae sunt actionis, inest quaedam vis a natura data; qua re etiam hac imperiti, hac vulgus, hac denique barbari maxime commoventur [...]. actio, quae prae se motum animi fert, omnis movet (, dabei wohnt auch all dem, was zum Vortrag gehört, eine gewisse naturgegebene Kraft inne. Deshalb werden auch durch sie die Laien, die breite Masse, ja selbst die Barbaren zutiefst beeindruckt. [...]. Der Vortrag, der die Regung des Gemüts zur Schau trägt, spricht sie alle an'). (Cicero, De oratore III 223).

18 isdem enim omnium animi motibus concitantur et eos isdem notis et in aliis agnoscunt et in se ipsi indicant (,denn aller Menschen Herzen werden von denselben Regungen bewegt, und an denselben Zeichen, die sie bei ihnen selbst bezeichnen, erkennen sie sie auch bei anderen').

19 Aus seiner romzentrierten Sicht trifft dies wohl schon zu, aber schon sein Hinweis auf die „üblichste Gebärde“ des alten Rom (XI 3, 92), die Schließung der Spitzen von Daumen und Mittelfinger bei nach oben offener Handhaltung, wurde in der folgenden zeitgenössischen Rezeption so nicht akzeptiert (Kalverkämper 1998b: 1346). 
,Sprechen` und ,Körperverhalten` schon aus ältesten Erfahrungen heraus miteinander verbunden sein müssen, eben weil sie beide die Aufmerksamkeit des Partners in Anspruch nehmen, also Rezeption und Reaktion steuern, läßt sich aus dem Indogermanischen ableiten: Lateinisch dicere ,verlautbaren ", , sagen " geht auf die indogermanische Wurzel *deik-, zeigen` zurück ${ }^{20}$.

3.2. Es verwundert vor diesem Hintergrund, der ja anthropologische Gegebenheiten - somit Fundamentales der conditio humana - spiegelt, $\mathrm{da} ß$ die Rhetorik sich hier nicht stärker systematisch engagiert hat und es nur bei kritischen Hinweisen insbesondere zu Stimme ${ }^{21}$, Mimik, Gestik und Gesamthaltung sowie zu kommunikativ wirkenden Attributen (z.B. Kleidung, Schmuck, Gegenstände wie [Redner-]Stab) beließ. Das Ungleichgewicht mag zum einen damit erklärt werden können, (1) daß die Sprach-Komponente der Rhetorik durch ihre Partnerinnen des Trivium gestützt wurde: Grammatica - die erste unter den artes - und Dialectica sind, als theoretische Künste ${ }^{22}$, deutlich wort- und textbezogene Disziplinen; (2) zum andern hat gerade die Antike eine mächtige Tradition theatralischer Praxis und zugehöriger literarischer Reflexion, und die Poetik ${ }^{23}$ ist natürlich eine Kunst der Sprache: der Dichter, griech. pointhvı poietés ,Macher', gilt als Wortkünstler, wobei er die Wirklichkeit in konzentrierter Weise (griech. kaqovlou kathólou) nachbildet (griech. m ìmhsiı mímesis). ${ }^{24}$ Mit der Aufführung beginnt die praktische Kunst. ${ }^{25}$ Die Kunstgattungen - die poietische (Poesie; auch Rhetorik bei den niedergeschriebenen Reden sowie mit ihren Strukturteilen inventio, dispositio und elocutio, die ja echte Sprach-Anteile sind), die praktische (Aufführung: Rhetorik mit

20 Griech. deiknuvnai deiknýnai, zeigen“; vgl. auch nhd. (jdn.) zeihen; nhd. zeigen.

21 Eine Kulturgeschichte der Stimme stellt Göttert (1998) vor, in der die Anteile der Rhetorik und des Theaters (Bühne) sowie der Predigt naturgemäß eine wichtige Rolle spielen, bis dann die Technik (Kabel, Lautsprecher) neue Wirklichkeiten schafft.

22 Griech. tevcnai qewrhtikai téchnai theoretikaí; lat. artes in inspectione positae.

23 Die poetischen Künste (griech. tevcnai poihtikai téchnai poietikaí; lat. artes in effectu positae ) mit dem höchsten Konkretheitsgrad als ,herstellende“ Künste: mit dem artifex ,Künstler' und dem opus ,(Kunst-) Werk'. (Vgl. Lausberg 1960: §10).

24 Vgl. Lausberg (1960: §35).

25 Griech. tevcnai praktikai téchnai praktikaí ; lat. artes in agendo positae, mit dem actor ,Schauspieler' und der actio ,Vortrag', ,Darstellung' des poietischen opus ,Werk'. 
ihren Teilen memoria und actio; Dialektik; Musik) und die theoretische (Grammatik; Arithmetik; Geometrie; Astronomie) - finden in der actio als Schnittpunkt zusammen ${ }^{26}$. Sie ist gleichsam der Brennpunkt, in dem sich die Künste in effectu, in agendo und in inspectione treffen, um auf das Resultat, das (sprachliche, poietische) opus, zu zielen.

Da die actio zwischenmenschlich, somit dialogisch verläuft - der eine agiert auf den Partner hin, der wiederum rezipiert das und reagiert daraufhin -, ist für das Gelingen des Kommunikationsprozesses eine prinzipielle Voraussetzung vonnöten, die als solche auch für alle artes gilt: nämlich das accomodate dicere, die Angemessenheit also; sie gilt in der Rhetorik als eine Tugend (virtus), die man - unter dem Stichwort aptum $^{27}$ - als Dichter, Schauspieler oder Rhetor zu beachten hat. Sie bezieht sich als Zweckhaftigkeit des Ganzen auf dessen Teile, nämlich auf den Rede-Inhalt, auf die Partner und auf die Situation hin; somit umgreift sie, wie Quintilian ausführt (XI 1,1-93), das Kunstwerk selbst (,,inneres aptum“) und seine soziale Einbettung (,,äußeres aptum“, das den Sprecher, das Publikum, den Zeitpunkt der Rede, den Ort der Rede berücksichtigt). Insbesondere für die Ausgestaltung der elocutio als Sprachfassung und der pronuntiatio als der vorgetragenen Präsentation ist das aptum wirkungsmächtiger Bestandteil, um das prinzipielle Selbstverständnis der alten Rhetorik - als ars bene dicendi - und um die Zielsetzung einer Rede, nämlich ihre Zweckhaftigkeit des Überzeugens bzw. Überredens der Zuhörerschaft (griech. peì qe in peíthein, lat. persuadere) durch die Gemeinschaft von docere 28 als dem intellektuellen Bestandteil (,Einsicht', ,Belehrung '), und von delectare (conciliare) (,Unterhaltung', ,Vergnügen') und movere (concitare) (,Gemütsbewegung') als den affektiven Komponenten zu gewährleisten. Allerdings spielte das äußere aptum für die Theoretiker keine eigenständige Rolle; sie verlegten sich eher auf Differenzierungen im Bereich der elocutio. ${ }^{29}$ Dies dürfte als die dritte Erklärungsmöglichkeit für das Fehlen einer rhetorischen Systematik des körpersprachlichen Einsatzes neben der Nähe zu den Sprach-artes - s.o. (1) - und den Anteilen an der poietischen ars - s.o. (2) - gelten.

\footnotetext{
26 Siehe Lausberg (1960: $§ 10,4)$.

27 Lausberg (1960: $\$ 256,258,1055-1062)$; andere Termini sind accomodatum (Quintilian VIII 1, 1), decens, decorum, griech. prevp on prépon.

28 Quintilian XII 10, 59. Auch probare, prodesse.

29 Siehe Lausberg (1960: §1058).
} 
3.3. Wie stark der Sprachbereich der elocutio interessierte, und zwar auf Kosten der actio-Anteile, läßt sich an dem Fehlen einer Situationstypologie ermessen. Das lag auch gar nicht so sehr auf der Hand, war doch die Situation, die pragmatisch vorhanden, gelebt, „gesprochen“ wurde, ebenjene konkrete, wie sie in loco ablief. Die rhetorische Situation genügte sich als Kategorie selbst. Wichtiger dagegen waren jene Situationen, über die berichtet, erzählt, verhandelt, nachgedacht, geurteilt wurde; sie aber spielten sich über die sprachliche Darstellung ab, als Abbilder der Wirklichkeit, und für sie standen dann, dem aptum gemäß, die Kategorien in hoher Differenziertheit zur Verfügung: Mit vier zentralen Fragen (quaestiones) nach dem status (,Stand' einer Situation, die in der Rede behandelt wird; Hauptproblem) wird erkundet (Quintilian III 6), welche soziokulturellen Umfelder - Handlungssituationen - gegeben waren. Der Redner stellt sich (1) die Vermutungsfrage (status coniecturae), mit der er die Frage klärt, ,ob der Angeklagte es getan hat'; dann (2) die Definitionsfrage (status finitionis), die beantwortet, ,was er getan hat'; anschließend (3) die Rechtsfrage (status qualitatis), die eine Tat abwägt, ob sie zu Recht vom Angeklagten getan worden ist; und schließlich (4) die Verfahrensfrage (status translationis), mit der geprüft wird, ob ein Verfahren überhaupt zulässig ist. ${ }^{30}$ Für die Beweisführung standen dann aus der argumentatioLehre ,Fundstätten“ für Beweise, die tovpoi tópoi, lat. loci, zur Verfügung, die ihrerseits eine recht dichte Strukturierung der Welt des Handelns vorstellen - geschieden nach personenbezogenen ( a persona) und sachbezogenen ( $a$ re) Aspekten, wobei letztere ein außerordentlich reiches Arsenal strukturierter Handlungswelt bieten. ${ }^{31}$ Neben der Gerichtspraxis hat sich die Status-Lehre auch für andere Gattungen bewährt, so bei den (juristisch affinen!) Konflikten des Dramas. ${ }^{32}$

Es liegt somit nahe, daß man hier eine antike Handlungslehre erkennen mag: Rhetorik und Poetik, insbesondere in der Lehre von den dramatischen Gattungen, als ,eine sehr differenzierte und teilweise höchst subtile Handlungstheorie“ (Weinrich 1976: 24) und somit als eine Pragmatik (Fuhrmann 1975). Für den genuinen Ort der Pragmatik aller-

30 Vgl. Lausberg (1960: $§ \S 79-138)$; Ueding / Steinbrink (21986: 28f., 237); Göttert (1991: 19-21); Kalverkämper (1999a).

31 Vgl. Lausberg (1960: $\$ \$ 373-399)$; Ueding / Steinbrink (21986: 218-235).

32 Lausberg (1960: § 96); griech. dra-n $d r A$ a n, handeln“, ,tun“ (ebenso wie griech. poiein poiein). 
dings, wo eine Handlungstheorie konkretisiert wäre, mit dem Körper als kommunikativem Mittel der Ausgestaltung von Inhalten - d.h. also: für die Beredsamkeit des Leibes als einem funktionalen Ausdrucksmittel in der Kommunikationssituation - hat die Rhetorik nur ein trübes Auge übrig.

Die actio wird sich mit der Zeit aus der eher sprachinteressierten Rhetorik weg dorthin weiterentwickeln, wo sie dann künstlerisch gebraucht und separat geachtet wird: bei der Theatralik ${ }^{33}$, bei der höfischen Zeremonialkultur des 16. bis 18. Jahrhunderts ${ }^{34}$, in der Kunst, hier besonders in der bildenden Kunst (Skulptur und Malerei), wozu oben (s. 1.) schon der Einsatz mit der Renaissance angesprochen und die scharfe Beobachtungs- und Reproduktionsleistung der italienischen Malerei hervorgehoben wurde. Eine Reflexion oder eingehendere Beachtung findet sich in den Rhetoriktheorien des ausgehenden 16. Jahrhunderts dementsprechend nicht mehr. ${ }^{35}$

Wenn nun aber das Funktionieren von Kommunikation darin besteht, daß die beteiligten Komponenten - von der ,Person' und ihrer ,Sprache' bzw. ihrem ,Sprechen-in-Texten-und-Situationen' bis zum ,Medium' und einer prinzipiellen ,Kultureinbettung' (s.o. 2.) - integriert sind, verbietet sich eine derartige Trennung in einen Bereich der ,Sprache" und einen des ,Handelns'. Auch im Prozeß der Verselbständigung der actio ist, wie man an den betroffenen Disziplinen sieht, der Sprachanteil immer mitgegeben, oder aber es handelt sich - wie in der Theatralik bei der Pantomime - um gattungsspezifische Reduktionen.

3.4. Eine Kulturgeschichte der actio muß noch geschrieben werden; dieses Desiderat würde auch disziplingeschichtlich - und dabei dann auch jeweils gattungssystematisch - auszulegen sein. Nach der Entdeckung des kommunikativen Körpers wäre also nun noch die Geschichte der Beachtung des kommunikativen Körpers zusammenhängend darzustellen; den letzten Zustand dieses Prozesses kann man in

\footnotetext{
33 Vgl. z.B. Barnett (1987); Pfaff / Keil / Schläpfer [Hrsg.] (1996).

34 Vgl. Hofmann (1985) und (1990); Kapp (1990).

35 Für die Sicherung dieser Einschätzung, die eingehende Beschäftigung mit den Rhetoriken der frühen Neuzeit voraussetzt, danke ich meinem romanistischen Kollegen Reinhard Krüger (Berlin). Der Befund scheint den historischen Gegebenheiten angemessener zu sein als die Darstellung von Göttert (1998), der in Kap. XI. („Die Beredsamkeit des Leibes“) eine „Rückkehr der actio“ behauptet.
} 
der aktuellen Zeit, seit den siebziger Jahren, mit wachsender Brisanz erfahren: nämlich die Wiederentdeckung des kommunikativen Körpers. Doch gerade hierbei fehlt noch ein ausgeprägtes Bewußtsein für die historische Kontinuation. Einzelne Inseln gezielten Forschungsinteresses - so, thematisch recht naheliegend, die frühe Neuzeit - sind inzwischen erarbeitet. ${ }^{36}$ Aber eine Kulturgeschichte der ,Leiblichkeit (wie es die Anthropologen ${ }^{37}$ und - das Thema seit einigen Jahren neu aufgreifend - die Literaturwissenschaftler ${ }^{38}$ formulieren) oder der ,Körperlichkeit‘ (wie es die Semiotiker und Künstler sehen) bzw. der ,Körpersprachlichkeit' (wie es die Linguisten und Kommunikationswissenschaftler ausdrücken) fehlt immer noch. ${ }^{39}$ Für die heutige Zeit steht der kommunikationswissenschaftliche Fokus als ,Körpersprache“ im Vordergrund des Interesses. 40

\section{Die Wiederentdeckung aus pragmatischen Zwängen: Interkulturelle Wirtschaftskommunikation}

4.1. Die Eigengesetze der Informations- und Kommunikationsgesellschaft, der Medien- oder (inzwischen) Multimediagesellschaft haben auch die länger schon bestehenden Etiketten von der Leistungsund der Konsumgesellschaft in die kritische Diskussion gezogen, wodurch wirtschaftliche Aspekte, gerade auch mit dem Fokus auf Konkurrenz, beherrschend wurden. Wirtschaft und Kommunikation waren seit jeher miteinander verflochten - der Begriff des Handels ist gar nicht anders denkbar. Die beginnende Internationalisierung der Handelsbeziehungen zu Anfang dieses Jahrhunderts hob die fremdsprachlichen Herausforderungen zur Wirtschaftskommunikation in den Blick und verlangte nach normsprachlich-terminologischen Regelun-

36 So z.B. Kapp [Hrsg.] (1990); Barnett (1987); Behrens / Galle [Hrsg.] (1993).

37 Insbesondere die Historische Anthropologie beschäftigt sich, schon zu einer Art Zentrum der Kulturwissenschaften avanciert, mit , Körpergeschichte‘, , Geschlechtergeschichte " und ,kulturellem Gedächtnis'.

38 Hinweise in Kalverkämper (1997) sowie in (1990) und (1991); Schmauser (1998); Behrens / Galle [Hrsg.] (1995).

39 Vgl. Kalverkämper (1997).

40 Sicherlich läge es auch nahe, die semiotische Sicht als die dominierende zu benennen; andererseits sei nicht verschwiegen, daß dort die nonverbale Kommunikation als solche im Blick ist und die sprachliche Seite nicht genügend einbezogen wird; mit dem ,Kommunikations'-Begriff dürfte dem integrativen Verhältnis, wie es ja auch das Modell (s.o. 2.) zeigt, aber entsprochen sein. 
gen. Die sogen. Wirtschaftslinguistik der dreißiger Jahre ${ }^{41}$ hat hier eine historiographisch wichtige Vorreiterfunktion innegehabt, die dann durch den Zweiten Weltkrieg abrupt abgebrochen wurde und sich ab den spätfünfziger, sechziger Jahren mit dem Aufkommen der Terminologieforschung ${ }^{42}$ und dann, in den siebziger Jahren, der Fachsprachenforschung, die heutzutage sich viel komplexer eher als eine Fachkommunikationsforschung versteht ${ }^{43}$, nicht in alter Kontinuität weiterentwickelt hat (Picht 1998: 340). Sie hat aber mit ihrer Bestimmung, daß die Wirtschaftslinguistik ,,der Völkerverständigung und darüber hinaus der Selbsterkenntnis“ (Messing 1932: 7) dient, ausdrücklichen Wert auf einen korrelativ begriffenen (,inter-'!) Kulturgewinn gelegt, der sich aus einer prinzipiell interdisziplinären Interessenlage (Wirtschaft und Kommunikation) speist. Das waren methodologische Positionen, die aus heutiger Sicht durchaus als interkulturell angesehen werden können, was schon damals deutlich über rein merkantile oder finanzielle Aspekte hinausreichte.

Heutzutage ist der partnerschaftliche und somit partnerachtende Aspekt des ,Inter-' prägend für das Selbstverständnis, das die beiden interdisziplinär kooperierenden Disziplinen pflegen, also die kulturwissenschaftlich und angewandt ausgelegte Linguistik und die an Sprache, wirtschaftsbezogenen Textsorten und Kommunikationsprozessen (z.B. Verhandlungen, Geschäftsabschluß, Kaufverträge, Gebrauchsanweisungen, Patentschriften, Planungspapiere, usw.) sowie an Mündlichkeit (Rhetorik, Textoptimierung, Körperverhalten, soziale Aspekte der Kommunikationssituation) interessierte Wirtschaft. Dieses gemeinsame Selbstverständnis bestimmt sich als Brückenschlag, mit einem seriösen Interesse an dem fremden Gegenüber mit seinen kulturbedingten Erwartungen, Kenntnisvoraussetzungen und Verhaltensspezifika und natürlich auch mit seiner fremden Sprache, der Achtung entgegenzubringen ist (und nicht selbstverständliche Sprachendominanz, z.B. des Englischen, oder Konkurrenzentscheidung gegen eine sogen. „kleine Sprache“). Mit dem Handel waren seit alters her die Interlingualität (Sprachenkontrast, fremde Sprachen, Notwendigkeit von Dolmetschen und Übersetzen bzw. von Fremdsprachenlernen) und

\footnotetext{
41 Zur Orientierung sei Picht (1998) empfohlen.

42 Vgl. Oeser / Picht (1998).

43 Vgl. Kalverkämper (1996).
} 
die Interkulturalität (Begegnung mit dem Fremden; Einstellung auf kulturell Unbekanntes; Eigenflexibilität bei der Fremdwahrnehmung; Vorurteil, Stereotyp, Klischee; Dialog-, Lern- und Korrekturbereitschaft; Prozesse der Adaptation von Fremdem, Nutzung von Chancen der eigenkulturellen Weitergabe, also letztlich Wege der gegenseitigen Bereicherung) verbunden, übrigens ebenso wie - gleichfalls bereits in historischer Zeit - mit dem Pilgerwesen ${ }^{44}$, den Eroberungskriegen ${ }^{45}$, den Entdeckungen und den Berichten darüber ${ }^{46}$, oder den Reisen ${ }^{47}$.

4.2. $\mathrm{Da}$ sich heutzutage das Bewußtsein so stark auf die Wirtschaft und die finanziellen Aspekte lenkt, hängt wohl mit dem dritten Faktor gleichsam als der Resultante zwischen Kommunikation einerseits und Handel oder Wirtschaft andererseits - zusammen, nämlich mit der telekommunikativen und wirtschaftlichen Globalisierung. Sie rückt das Aufeinanderprallen fremder Sprachen und kulturbedingter Verhaltensweisen so eng aneinander, daß Konflikte daraus sich kaum mehr ,,aufbauen“, vielmehr fast direkt vorhanden sind. Und der dafür notwendige Bedarf an Vermittlung, speziell in Gestalt von Translation (Dolmetschen, Übersetzen) zwischen Eigenem und Fremdem meldet sich so drängend und rasch, daß gegenüber der Fremdkultur gewisse Gewöhnungen, vorsichtiges Herantasten, pragmatisches Erfahren, also ein Aufbau sensibler Empirie der Fremdkulturalität selten oder sogar praktisch ausgeschlossen ist. In einer ,globalisierten“ Gesellschaft ist die Überbrückung von Grenzen, wie sie durch die Verschiedenheit der Sprachen und Verhaltensweisen nun einmal gegeben sind, eine maßgebliche Voraussetzung; jedenfalls wirken sich Kommunikationskonflikte, Verhaltensbarrieren, kulturell verankerte Unzugänglichkeiten nicht befördernd auf Kooperation und Integration aus.

Die Wissensgesellschaft, wie sie nun auch abstrakt nach ihren primären und wirkungsmächtigsten Intentionen bezeichnet wird, braucht

44 Z.B. europäische Pilgerstraßen wie die nach Santiago de Compostela.

45 Man denke nur an die enorme Wirkung der Alexander-Feldzüge im Altertum, an die Kreuzzüge im Mittelalter, an die Expansion der Araber nach Spanien oder an die osmanischen Westausdehnung bis vor Wien.

46 Erinnert sei nur an Marco Polo oder an Kolumbus.

47 Erwähnt seien die in Mittelalter und Renaissance zunftgeforderten Wanderschaften von Künstlern in fremde Länder zur Bereicherung des eigenen Wissens, oder die berühmte Seidenstraße wie auch die Salzstraße, oder technisch-moderner, z.B. die Transsibirische Eisenbahn. 
Formen der Überbrückung, der Vermittlung derartiger Anforderungen, und sie sucht diese in der Antizipation; deren fundierteste Art das Lernen von fallbezogenen Strategien und Kategorien und somit das Wissen um mögliche Erscheinungs- und Bewältigungsweisen ist. In der sich zunehmend international verflechtenden Wirtschaft wird das Bewußtsein um partnerbezogene Dialogbereitschaft, die auch die Kulturalitätsaspekte umfaßt, besonders geschärft, entscheiden sich doch gerade über diese Dimension Sympathie und Antipathie, Gelingen oder Mißlingen von Vertragsabschlüssen, Aufbau und Bestand oder aber Zusammenbruch von Kontakten, somit dann auch finanzieller Erfolg und Wachstum oder Einbuße und Fehlinvestition.

4.3. Die Unternehmen haben dies als marktwirtschaftlich sensiblen Aspekt ihres Geschäftserfolgs und ihrer Corporate Identity erkannt; in den neunziger Jahren ist das Interesse der Wirtschaft, der Industrie, auch der angewandten Forschung an Ausbildung ihrer Führungskräfte insbesondere auf der Ebene des gehobenen Managements zu ebendiesen Problemkreisen um Interkulturalität gewachsen. Das körperliche Verhalten in Kommunikationsprozessen wurde zum Thema, als die internationale Wirtschaftsverflechtung die Kulturunterschiede überaus deutlich als potentiellen Störfaktor zutage treten ließ. Finanzieller Profit war ja immer schon der Motor - im wahrsten Sinne: ,Beweggrund ' - für die Besetzung neuer Themen (und seien sie ihrerseits noch so alt) und für eine intensive Beschäftigung mit ihnen. Da es um hohes Prestige und finanzielle Konsequenzen ging (und geht), bei denen Faktoren wie zwischenmenschliche Sympathie, fremdsprachliches Verstehen, kulturenkontrastives Verständnis, partnergerichtete Achtung eine nicht zu unterschätzende, oft ausschlaggebende Rolle spielen, begannen verschiedene (Groß-) Unternehmen in den achtziger Jahren, im eigenen Hause Schulungskurse durchzuführen; inzwischen bieten auf dem freien Markt auch Service-Firmen für Unternehmensberatung Seminare zum interkulturellen Handeln in der Wirtschaft - dies unter dem Begriff Cross Culture - an ${ }^{48}$; Angewandte Rhetorik, Verhandlungsrhetorik, findet sich als Ausbildungsschwerpunkt in eigens auf internationale Unternehmenskommunikation ${ }^{49}$ hin ausgerichteten Trainingslehrgängen. Der Begriff der ,Unternehmenskultur', der in den

48 Vgl. z.B. Bolten [Hrsg.] (1995).

49 Vgl. z.B. Bolten [Hrsg.] (1998). 
neunziger Jahren bei den international tätigen Konzernen aufgekommen ist, umgreift inzwischen fest die Ausbildung eines kulturensensibilisierten Managements. ${ }^{50}$

In jener Zeit pragmatisch erzwungener unternehmerischer Selbstinitiativen etablierte sich, über die Fachsprachenforschung (Wirtschaftssprache) einerseits und die Interkulturelle Germanistik (Interkulturalität) andererseits nahegelegt, gleichsam begleitend die universitäre Forschungsrichtung, Interkulturelle Wirtschaftskommunikation “51. Als wissenschaftliche Inter-Disziplin legt sie, wie auch die Schulungen in der Praxis bei ihren prinzipiell notwendigen Lehrinhalten, Wert darauf, daß neben der

- sprachlichen Textschulung (die der elocutio des rhetorischen Systems entspricht ${ }^{52}$ ) unter medialen Aspekten (Mündlichkeit, Schriftlichkeit) und varietätenbezogenen Gesichtspunkten (Fachsprache, Texte für Fachleute [Wirtschaftswissenschaftler, Unternehmer], Texte für Fachmann-Laie-Kommunikation [Beratung, Politik, Journalismus, Sachbuch, Wirtschaftssendung im Fernsehen, u.a.]) auch

- Persönlichkeitsformung des einzelnen Entscheidungsträgers (Bewußtheit, Eigenwertigkeit, Stabilität, Körperbeherrschung) und

- Bewußtmachung von Kulturspezifika des fremdkulturellen Partners analysiert und gelehrt werden.

$\overline{50}$ Als ein Beispiel (neben vielen) für eine Buchversion sei auf Rowland (1994) hingewiesen.

51 Orientierung z.B. bei Müller [Hrsg.] (21993).

52 Die elocutio (griech. levxił w wravsiı léxis, phrásis ) ist der sprachliche Ausdruck. Zu ihr gehören als Vertextungstugenden (virtutes):

- die idiomatische fehlerfreie, grammatisch einwandfreie Korrektheit (puritas);

- die Transparenz und Verständlichkeit des Gesagten (perspicuitas);

- eine stilistisch-ästhetisch angenehme Präsentation (Schönheit der Rede, ornatus), was mit einem überaus reichen Arsenal an Kunstgriffen systematisiert ist, und wozu auch neben einer hochdifferenzierten Figurenlehre die Anweisungen für geschickte Komposition gehören; schließlich

- die situationsspezifische Angemessenheit des Gesagten (aptum, das sich mißt an der Angelegenheit, die zur Beredung ansteht [utilitas causae] und auf den Rede-Erfolg zielt, der in der Überredung besteht und darin seinerseits wiederum von Verständnis und Meinung des Publikums bzw. Partners abhängt).

Lausberg (101990: 3. Teil); Ueding / Steinbrink (21986: 199-214, 264-303); Kalverkämper (1999a). 
4.4. Die Rhetorik dient hier als die verbindende Klammer, denn ihr Format ist ja geradezu dafür angelegt, Sprache (Text, Rede) und Eigenwirkung und Fremdeinschätzung unter dem Gesichtspunkt der Überzeugung und somit der Erfolgsgewinnung und Erfolgssicherung effizient zu verflechten. Und so rücken die konkreten - mündlichen, dialogischen - Sprachverwendungssituationen von Konferenz, Management, Verhandlung, Vertragsabschluß u.a. in den Blick, als pronuntiatio mit dem nun wichtigen Bereich der actio, wodurch das körpersprachliche Verhalten wiederentdeckt wird und in - allerdings immer noch mehr oder weniger bescheidenem Rahmen (s.u.) - eigen- und interkulturell bewußt trainiert wird, mit dem Ziel, die jeweiligen Fremderwartungen und Fremdwahrnehmungen antizipieren zu können, damit sich so eine optimierte Wirtschaftskommunikation über Sprachen- und Kulturengrenzen hinweg möglicherweise ergibt. Dieses ideale Ziel wird allerdings, schaut man sich die Printmedien, Schulungsvideos und Lehrhinweise genauer an, eher nicht thematisiert; vielmehr beherrschen Strategien für Effizienzsteigerung, das partnerachtende Sich Durchsetzen, der wirtschaftliche Erfolg, wie er sich z.B. im Verhandlungsergebnis, im Vertragsabschluß, in der internationalen Kontaktpflege zeigt, also das Maß einer direkten Pragmatik, die Absichten, Körperkommunikation und ihren kulturspezifischen Stellenwert zu berücksichtigen: die Rhetorik der Macht ${ }^{53}$, des Expertentums ${ }^{54}$ und der Werbung ist hier gefragt. 55

Die Wiederentdeckung des kommunikativen Körpers, wie sie insbesondere über die Bedürfnisse der Interkulturellen Wirtschaftskommunikation verläuft, verhilft allerdings zur Zeit nicht dazu, die Diskussion um Körperkommunikation und Körpersemiotik wissenschaftlich verwerten zu können. Indem

- die Kriterien zur Analyse weitgehend fehlen,

- die Ganzheitlichkeit des Ausdrucksrepertoires nicht oder nur unzureichend beachtet wird und

- sehr großer Wert auf Verhaltensoptimierung in Bezug auf den Part-

53 „Körpersprache der Bosse“ (Schwertfeger / Lewandowski 1990); „Körpersprache für Manager“ (Rückle $\left.{ }^{8} 1991\right)$, u.a.

54 Sehr gute Analyse von „Expertenattributen“ bei Lotte Weinrich (1992: Kap. 3.5.2.); dazu auch Kalverkämper (1994: 139-143). Vgl. auch Bentele / Hess-Lüttich [Hrsg.] (1985).

55 Kalverkämper (1998b: 1362f.). 
ner gelegt wird (was auch eine Auswirkung auf die wirtschaftlichen Ergebnisse im internationalen, interkulturellen Verkehr mit sich bringt),

ist eine eigenständige Erforschung der Körperkommunikation von hier nicht in Sicht. Vielmehr spielt in der Interkulturellen Wirtschaftskommunikation das analytische Interesse an der (Fach-) Sprache, an den schriftlichen Textsorten und an den Texten und ihren sprachlichen Phänomenen eine weitaus größere Rolle als die wissenschaftliche Beschreibung der körperbezogenen Spezifika. Diese auffallend ungleich verteilte Aufmerksamkeit liegt offensichtlich darin begründet, daß

- zum einen die Mündlichkeit immer noch gegenüber der Schriftlichkeit von fachlicher, auch von wirtschaftlicher Kommunikation 56 weniger Beachtung genießt, und dies nicht zuletzt wegen der unvergleichlich höheren Komplexität mündlicher Kommunikationsprozesse in Situationen, wobei die Körpersprache eben nicht nur einfach als solche Erwähnung finden sollte, sondern auch in

a den beteiligten Körperteilen - insbesondere Gesicht [Mimik], Arme mit Händen und Schultern [Gestik], die bewegte kommunikative Körperhaltung [Kinesik], das Nähe- bzw. Distanzverhalten [Proxemik] betreffend -,

a den Äußerungsabläufen mit ihnen,

a den kommunikativen Funktionen,

a den kulturspezifischen Bedingtheiten,

a den möglichen Partner-Interpretationen und -Reaktionen, seien diese verbal oder nonverbal,

mitgeteilt und als ganzheitliche Orchestrierung dargestellt werden müßten. Doch die Verkaufsrhetorik wirkt in den gedruckten Handreichungen (Bücher) gleich behandelt wie Rhetorik am Telephon ${ }^{57}$, der Körper als Kommunikationsfaktor, der neben dem gesprochenen Text mitbeteiligt ist am partnergerichteten Geschehen, scheint

56 Eine der jüngsten interlingualen Publikationen zur Wirtschaftskommunikation bestätigt wieder einmal diese Einschätzung: Padilla Gálvez [Ed.] (1998).

57 Vgl. z.B. Hofmeister (1990), ausdrücklich mit (u.a.) ,Körpersprache‘ als rhetorischem Lehrstoff auf dem Buchdeckel werbend, aber ohne eigene Lehreinheit und nur in verstreuten Zusatzhinweisen (s. Register) immer mal wieder auf deren Wichtigkeit hinweisend. - Oder Ebeling (91990: 206-221). 
gern noch vergessen zu werden oder nur einen kurzen Seitenblick eingeräumt zu bekommen;

- zum andern generell (!) das (linguistische, rhetorische, soziopsychologische, kulturwissenschaftliche, anthropologische) Analyse-Instrumentarium für die wissenschaftliche Erfassung von (kulturspezifischer) Körpersprache

a noch nicht ausgereift und noch $\mathrm{zu}$ grob ${ }^{58}$, in manchem dann wieder viel zu detailliert ${ }^{59}$, außerdem auch

a noch nicht genügend interdisziplinär ausgelegt ist und somit

a für eine Anwendung oder Lehre noch recht unpraktikabel vorliegt.

Die Balance zwischen Ganzheitlichkeit und Segmentierung, zwischen komplexem Beschreibungsarsenal und Detail ist immer noch nicht gefunden, und die Altmeisterin der Disziplinen, die Rhetorik, hat hier nicht die geeigneten Grundlagen gelegt (s.o. 3.).

Die Wiederentdeckung des kommunikativen Körpers verläuft eher über eine Art Anweisungsrhetorik, die Regeln und Rezepte bereithält und eine reiche Kasuistik (Fallbeispiele und ihre Diskussion mit dem Ziel des Bessermachens) vorstellt. Die Zielgruppe solcher Bücher (Printmedien) und Seminarthemen (Mündlichkeitstraining) (s.o. 4.3.) sind publikumsbezogene, öffentlichkeitsnahe Berufe (Lehrer, Prediger, Politiker) wie auch - unter den bereits erwähnten (s.o. 4.2.) wirtschaftlichen Gesichtspunkten von Effizienzsteigerung - Manager und Führungspersönlichkeiten. 60

Auch hier wäre wieder zu unterscheiden, ob es sich um Kommunikationsabläufe in der eigenen Kultur oder in einer Fremdkultur handelt. Die Angaben zu körpersprachlichen Signalen halten sich in solchen Populärrhetoriken 61 oder in den „Länderknigge“-62 oder „Kultur-

\footnotetext{
58 Vgl. z.B. Wallbott (1998).

59 Vgl. Frey (1984).

60 Z.B. Bierach (1996), Molcho (1997), Rückle ( $\left.{ }^{8} 1991\right)$.

61 Als nur ein Beispiel: Allnach / Rusch (1995), die ausdrücklich mit ,Körpersprache“ im Untertitel werben; tatsächlich aber finden sich in dem über 400 Seiten starken Buch unter dem Kapitel „Das Einüben der Rede“ nur drei Seiten zu dem Thema „Körpersprache und Mienenspiel“" (171-173); weiteres bei Kalverkämper (1995: 165-167). 62 Z.B. Rowland (1994). S. auch Kalverkämper (1994).
} 
Schock"-63Anleitungen oder berufs-, d.h. praktisch immer: wirtschaftsbezogenen Büchern ${ }^{64}$ quantitativ in Grenzen, sind dabei sehr heterogen, gehen meist als Rezepte vor (,wenn - dann" 65 ) und helfen in den vielfältigen Kontexten und Situationen des wirklichen Lebens dann doch nicht weiter. ${ }^{66}$

Derartige Populärdarstellungen unterschiedlichsten Niveaus zur Körpersprache füllen den Freibereich wissenschaftlich-methodologischer Zurückhaltung und haben inzwischen hohe Konjunktur: die Inspektion des Menschen ist des Menschen höchstes Interesse. Das treibt dann oft genug seltsame Blüten, die an - auch aus der Mentalitätsgeschichte her bekannte ${ }^{67}$ - geheime Wünsche erinnern lassen, „,hinter die Stirn“, ,in den Kopf“, „,ins Hirn“, „,in die Seele“ oder „,ins Herz" schauen zu können,

- um die letzte Ehrlichkeit ergründen zu können und somit sich ganz sicher zu sein - alles durchaus verständliche Sicherungs-Aspekte gerade auch der Wirtschaft, erst recht dann, wenn man mit Vertretern aus Fremdkulturen Vertrauen und Handlungssicherheit schaffen will; oder auch

- um selbst Macht ausüben und damit beruflichen Erfolg erreichen zu können - eine Art von theatralischer Selbstinszenierung - sowie

- um jene Partner, die als Vorgesetzte oder Autoritäten sozial höheren Rang innehaben (oder beanspruchen), besser in ihren Verhaltensstrukturen durchschauen und gegebenenfalls darauf reagieren $\mathrm{zu}$ können. 68

63 Wieder nur als Beispiele aus der großen Auswahl: Axtell (1989), Kalmbach (1990). Derartige Bücher verbinden ihr Anliegen auch vorzugsweise mit Aufklärung für Reisende, als fremdkultur,,bewußte“ Reiseführer (vgl. Kalverkämper 1995: 169-171).

64 Manager, „Bosse“, etc.: z.B. Rückle ( $\left.{ }^{8} 1991\right)$, Schwertfeger / Lewandowski (1990).

65 Als nur ein illustrierendes Beispiel unter erstaunlich vielen und dabei zum Thema ,Körpersprache' gleichartig vorgehenden, wenngleich vielfach in der Interpretation der Phänomene divergierenden Populärrhetoriken: Hofmeister (1990: 38-41): „Wenn plötzlich der Gesprächspartner [und nun folgt eine Liste von 49 Körperaktionen wie z.B. ,den Kopf ruckartig zurückwirft“ oder ,,die Brille hochhebt“" oder ,,mit den Händen ein Spitzdach formt“ oder ,die Füße ums Stuhlbein legt“] - Dann bedeutet dies [und wie im Vokabelheft findet sich jeweils gegenüber die semantische Auflösung wie „Ablehnung“, „Arroganz“, „Unsicherheit“ u.a.].“ Vgl. auch Kalverkämper (1995).

66 Vgl. die kritische Zusammenstellung in Kalverkämper (1994).

67 Man denke nur z.B. an die Physiognomik des 18. Jahrhunderts (und ihre Tradition aus der Antike) (Kalverkämper 1998b).

68 Vgl. z.B. Fast (1988) oder Schwertfeger / Lewandowski (1990). 
Doch umgekehrt gesehen schaden derartige Machwerke 69 einer seriösen interdisziplinär und interkulturell ausgelegten Erforschung der Körperkommunikation ${ }^{70}$, da sie Rezepte anbieten, mit der Aufdeckung des geheimen Körpers den Partner ,durchschauen“ und sich selbst entsprechend „wappnen“ zu können: Entlarvung von Fremdinszenierung und Tarnung als Eigeninszenierung sowie dann wieder die Tarnungsentlarvung schaffen hier problematische Weisen des Umgangs miteinander, die seriöse kommunikative Grundlagen wie ,Verstehen ', ,Dialogizität', ,Reaktionserwartung', ,Fremdkulturwahrnehmung' sowie die Palette der bekannten Griceschen Kommunikationsmaximen hintergehen und so gegen das humanum von Kommunikation überhaupt verstoßen, zumindest dazu verleiten, es außer Geltung zu setzen.

\section{Körper - Kommunikation - Kategorien: Ausblick}

Das Thema ,Körperkommunikation“ muß, so sollte evident geworden sein, als ein wichtiges Problemfeld der Internationalen Wirtschaftskommunikation schärfer erkannt und dann, in Ergänzung der bisherigen schriftbezogenen Text- und Lexik-Untersuchungen, nun auch im Bereich der situativen Mündlichkeit und Körpersemiotik wesentlich besser erarbeitet werden. Dazu wären folgende Eckforderungen zu berücksichtigen:

- Interdisziplinäre Annäherung und methodische Kooperation, was nicht die Wissenschaftsgrenzen aufheben soll, sie aber über die vorhandenen Verfestigungen hinaus öffnet und zu Grenzüberschreitungen zu gegenseitigem Erkenntnisgewinn einlädt ${ }^{71}$;

- Interkulturelle Dimensionierung, die voraussetzt, daß der Kulturenkontrast Unterschiede wie aber auch Gemeinsamkeiten zwischen der Fremdkultur und der Eigenkultur - die ihrerseits natürlich ebenfalls zu kennen wäre - berücksichtigt ${ }^{72}$;

69 Vgl. Kalverkämper (1995: 167f.).

70 Körpersprachenforschung, Körpersemiotik. - Es sei hier auf die Buchreihe Körper - Zeichen - Kultur / Body - Sign - Culture (Berlin: Berlin Verlag [Arno Spitz] 1998 ff.) hingewiesen, die sich u.a. ausdrücklich das Ziel setzt, solchen Tendenzen mit wissenschaftlichen Forschungsarbeiten entgegenzuwirken (hrsg. von Hartwig Kalverkämper / Reinhard Krüger / Roland Posner).

71 Zum Problem der Interdisziplinarität vgl. summarisch Kalverkämper (1998c: 1214).

72 Zum Problem der Interkulturalität vgl. die Aufarbeitung in Kalverkämper (1998a). 
- Bereitstellung eines seriösen funktionalen Analyse-Instrumentariums, das in einem kommunikativen Rahmen (dies wäre die Voraussetzung!) die Körpersegmentation (Ersehenskompetenz) und die ganzheitliche Interpretation (Bedeutungszuweisung) leistet;

- Umsetzung der wissenschaftlichen Erkenntnisse in die praktische Anwendung: Eine Kultureme-Didaktik 73 müßte erstellt werden, um die Fragen geeigneter Vermittlung von - im vorliegenden Fall Körpersprache in der Eigenkultur und in der Fremdkultur beantworten zu können; was bislang vorliegt, sind Zeugnisse von Dilettantismus, von längerer Lehrerfahrung im Unterricht, von geschulter Intuition, vereinzelt sicherlich auch von hervorragender Beobachtungskunst. Für die Lehre bedeutet dieser Zustand, daß Körpersprache einen eigenen Stellenwert bei der Vermittlung von Fremdsprachen und von muttersprachlicher wie auch fremdsprachlicher Fachkommunikation erhalten muß. Die bisher angebotenen Sprachkurse für Wirtschaftskommunikation genügen allerdings diesem Anspruch keinesfalls, und eine kritische Prüfung unter dem Aspekt ,Körpersprache' wäre auch als Angebot für eine sensibilisierende Lehrwerksoptimierung ${ }^{74}$ sicher nur zu begrüßen. ${ }^{75}$

Das Format der rhetorischen Wirklichkeit ist so weit (nämlich bis in die Kultur-Dimension hinein) und so komplex ausgelegt (nämlich von der Einzelperson bis zur Gemeinschaft reichend, von der Situation bis zur Kultureinbettung gespannt, Verbalität und Nonverbalität gleichermaßen umfassend), daß die wissenschaftliche Analyse, wenn sie sich einen Teilbereich, hier: die Körpersprache, herausnimmt, methodologisch dennoch die Isolation vermeiden und die Komplexität nachstellen muß: durch Ganzheitlichkeit, die sich mit integrativen Konzeptionen erreichen läßt. Das Problem ist in der Tat die Bewältigung der Komplexität.

\footnotetext{
73 Vgl. hierzu Kalverkämper (1995) und (1998b: 1341f., 1351f.).

74 Dies wäre ein attraktiver Zugewinn für das bisherige Aufgabenprofil der Sprachlehrforschung, wobei die Aspekte der Fremdsicht auf das Eigene - also Deutsch als Fremdsprache, English as a Foreign Language, Français Langue Étrangère, usw. - mit einfließen müssen und darin dann auch diese genuin kulturenkontrastiven Disziplinen bereichern.

75 So wäre z.B. zum „,Videosprachkurs für Wirtschaftsdeutsch“ (vgl. Krause / Bayard 1991) aus einer körperkommunikativen Sicht der Sprachlehrforschung etliches Kritisches anzuführen.
} 
Was jeder Mensch im mündlichen Kommunikationsprozeß leistet, nämlich die kommunikativen Signale des Partnerkörpers (1) zu erkennen und (2) zu interpretieren (sowie - (3) - eigene relevante Körpersignale auszusenden), kann eine Disziplin allein kaum analytisch erarbeiten. Um diese großartige Ersehens- (und Sende-) Kompetenz in ihren Elementen und in deren ganzheitlichem Funktionieren (auf eine kommunikative Eindrucks- bzw. Ausdrucksqualität hin) zu erfassen, bedarf es eines interdisziplinären Ansatzes, an dem die Anthropologie, Soziologie, Psychologie, Semiotik, Kommunikationswissenschaft, Linguistik und Rhetorik ihren jeweiligen Möglichkeiten entsprechend kooperieren, vielleicht - zu dieser Thematik - im umgreifenden Sinne einer „kommunikativen Kulturwissenschaft“.

Umsetzungsmöglichkeiten ihrer einschlägigen Erkenntnisse sind vor allem in der Internationalen Wirtschaftskommunikation gegeben, ja geradezu willkommen. Der Bedarf an (1) gesicherter Forschung und dann an (2) Strategien der Anwendung wissenschaftlicher Ergebnisse hat sich drängend aus den einschlägigen Beobachtungen der Wirtschaft ergeben, die die Defizite und Kommunikationskonflikte, die fremdkulturellen Unbedarftheiten und die (fremd-) sprachlichen Inkompetenzen als Hemmnisse für Markterschließung, für Standortfestigung durch fremdkulturelle Integration, für wirtschaftliches Florieren vor Ort und international, für Prosperität und Corporate Identity erkannt haben und nach effizienter Abhilfe suchen - bei der Rhetorik, Kommunikationswissenschaft und Linguistik, bei der Psychologie, bei den Theaterpraktikern (Schauspieler, Pantomimen). Bislang hat, wie dargestellt (s. 3.), nur die Rhetorik das weiteste Format an Beobachtungen und praktischen Hinweisen anzubieten, gerade auch unter den Aspekten der Wirkung und der Steuerung, die für die moderne Wirtschaftskommunikation wichtig sind.

Vor diesem Hintergrund wird die Rhetorik - bei aller (dargestellten) Beschränkung - eine zentrale Rolle spielen, wenn es um Körpersprache in Kommunikationsprozessen, um die actio geht; andererseits ist aus der Interkulturellen Wirtschaftskommunikation mit ihren besonderen Bedarfslagen (für die lehrende Umsetzung und für die Anwendung in den Verhandlungen und Wirtschaftsgesprächen) ein neuer Schub an Impulsen entstanden, der auf die Rhetorik und die Erforschung wirkmächtigen Redens und Schreibens in anderen Disziplinen fordernden Einfluß nimmt: hin 
- zu einer Systematisierung,

- zu ganzheitlich orientierten Analysen der Funktion von (kommunikativ eingesetzten Körper-) Teilen,

- zu situationstypologisch abhängigen Strategien,

- zu einem kohärenten Analyse- und Beschreibungs-Instrumentarium,

- zu einer wissenschaftlichen Methode der Komplexitätsbewältigung (z.B. durch Formen kriteriengestützter Reduktion, durch Relevanzsetzungen, durch erarbeitete Hierarchien, u.a.).

Ein solches Programm, das noch ausgebaut werden muß und hier lediglich in seinen Tendenzen als Punkte skizziert ist, kann von der modernen Rhetorik allein nicht bewältigt werden. Möglicherweise und dies wäre ein weiterer Grund für das Fehlen einer rhetorischen Systematik zum expressiven Körperverhalten (s.o. 3.2.) - haben die Theoretiker der Rhetorik dies ja gar nicht leisten wollen, gleichsam aus höherer Einsicht in das Unvermögen (oder sogar in die Unmöglichkeit), die permanente Präsenz des körperlichen Ausdrucksverhaltens begrifflich-sezierend zu erfassen. Auch ihnen damals war wie uns heute bewußt, daß es unmöglich ist, Körpersprache zu ignorieren und sie nicht zu interpretieren ${ }^{76}$; die lange Tradition der segmentierenden und funktionalen, also wissenschaftlichen Betrachtung des kommunikativen Körpers von seiner Entdeckung an bis zu seiner Wiederentdeckung legt es nahe, auch über die bisherige Unmöglichkeit nachzudenken, die Körpersprache angemessen zu beschreiben, zu strukturieren, zu systematisieren, in ihrem funktionalen Einklang und in ihren Aussagewerten (Bedeutungen) wissenschaftlich zu erfassen. In diesem Nachdenken liegt dann aber auch der Reiz (oder die Verpflichtung) beschlossen, die anspruchsvolle Aufgabe wissenschaftlich anzugehen, sonst schafft sich der Bedarf, wie kritisch vorgestellt (s.o. 4.3. und 4.4.), seine problematischen Nischen-Lösungen.

76 Kalverkämper (1995:143; 1997: 219-221), in analoger Erweiterung der bekannten Watzlawick-These, daß es unmöglich ist, nicht zu kommunizieren. 


\section{Literaturverzeichnis}

Allnach, Konstanze / Rusch, Caroline (1995). Rhetorik. Erfolgreiche Gesprächsführung - Redetechnik und Körpersprache - mit Übungen und Musterreden. München: Compact.

Axtell, Roger E. (1989). Vorsicht Fettnäpfchen. Fremde Länder, andere Sitten. Frankfurt: WDV Wirtschaftsdienst. (21991).

Barnett, Dene (1987). The Art of Gesture: The practices and principles of $18^{\text {th }}$ century acting. With the assistance of Jeanette Massy-Westropp. Heidelberg: Winter. (= Reihe Siegen. Beiträge zur Literatur- und Sprachwissenschaft. 64).

Behrens, Rudolf / Galle, Roland [Hrsg.] (1993). Körperbilder, Rhetorik und Anthropologie im 18. Jahrhundert. Würzburg: Königshausen \& Neumann.

Behrens, Rudolf / Galle, Roland [Hrsg.] (1995). Menschengestalten. Zur Kodierung des Kreatürlichen im modernen Roman. Würzburg: Königshausen \& Neumann.

Bentele Günter / Hess-Lüttich, Ernest W. B. [Hrsg.] (1985). Zeichengebrauch in Massenmedien. Zum Verhältnis von sprachlicher und nichtsprachlicher Information in Hörfunk, Film und Fernsehen. Tübingen: Niemeyer. (= Medien in Forschung + Unterricht. Serie A. Band 17).

Bierach, Alfred (1996). Körpersprache erfolgreich anwenden und verstehen. München: Südwest.

Bolten, Jürgen (1998). Fachsprachliche Phänomene in der Interkulturellen Wirtschaftskommunikation. In Hoffmann, Lothar / Kalverkämper, Hartwig / Wiegand, Herbert Ernst [Hrsg.] (1998). Fachsprachen / Languages for Special Purposes. - Ein internationales Handbuch zur Fachsprachenforschung und Terminologiewissenschaft I An International Handbook of Special-Language and Terminology Research. Band 1, Art. 88. Berlin - New York: de Gruyter. (= HSK - Handbücher zur Sprach- und Kommunikationswissenschaft. 14, 1). 849-855.

Bolten, Jürgen [Hrsg.] (1995). Cross Culture - Interkulturelles Handeln in der Wirtschaft. Jena: Wissenschaft und Praxis. (= Schriftenreihe Interkulturelle Wirtschaftskommunikation. 1).

Bolten, Jürgen [Hrsg.] (1998). Studien zur internationalen Unternehmenskommunikation. Leipzig: Popp. (= Angewandte Wirtschaftslinguistik. 1).

Cicero, Marcus Tullius (21981). De oratore - Über den Redner. [Entstanden 55 v.Chr.]. Lateinisch - deutsch. Übers. u. hrsg. v. Harald Merklin. ${ }^{2}$ durchges. u. bibliograph. erg. Stuttgart: Reclam.

Cicero, Marcus Tullius (21980). Orator. [Entstanden 46 v.Chr.]. Lateinisch - deutsch. Hrsg. v. Bernhard Kytzler. ${ }^{2}$ München: Heimeran.

Curtius, Ernst Robert (1938). Zur Literarästhetik des Mittelalters. I, II, III. In Zeitschrift für romanische Philologie 58, 1-50, 129-232, 433-479.

Curtius, Ernst Robert ( $\left.{ }^{7} 1969\right)$. Europäische Literatur und lateinisches Mittelalter. Bern München: Franke. 
Dubois, Jacques / Edeline, F. / Klinkenberg, J. M. / Minguet, P. / Pire, F. / Trinon, H. (1970). Rhétorique générale. Paris: Larousse. - Dt. Übers.: Allgemeine Rhetorik. Übers. u. hrsg. v. Armin Schütz. München: Fink 1974.

van Dülmen, Richard [Hrsg.] (1998). Erfindung des Menschen. Schöpfungsträume und Körperbilder 1500 - 2000. Wien - Köln - Weimar: Böhlau.

Ebeling, Peter ( $\left.{ }^{9} 1990\right)$. Rhetorik. Wiesbaden: Englisch.

Fast, Julius (1988). Körpersignale der Macht. Der kreative Weg zu mehr Erfolg und Einfluß. München: Heyne.

Frey, Siegfried (1984). Die nonverbale Kommunikation. Stuttgart: SEL-Stiftung für technische und wirtschaftliche Kommunikationsforschung im Stifterverband für die Deutsche Wissenschaft.

Fuhrmann, Manfred (1975). Die linguistische Pragmatik und die rhetorische StatusLehre. In Weinrich, Harald [Hrsg.] (1975). Positionen der Negativität. München: Fink. 437-439.

Göttert, Karl-Heinz (1991). Einführung in die Rhetorik. Grundbegriffe - Geschichte Rezeption. München: Fink. (= Uni-Taschenbücher. 1599). - (21994).

Göttert, Karl-Heinz (1998). Geschichte der Stimme. München: Fink.

Gumbrecht, Hans Ulrich / Pfeiffer, K. Ludwig [Hrsg.] (1988). Materialität der Kommunikation. Frankfurt/M.: Suhrkamp. (= Suhrkamp Taschenbuch Wissenschaft. 750).

Hofmann, Christina (1985). Das spanische Hofzeremoniell von 1500 - 1700. Frankfurt Bern: Lang.

Hofmann, Christina (1990). Das Spanische Hofzeremoniell - eine spezifische Ausdrucksform nicht-verbaler Sprache. In Kapp [Hrsg.] (1990: 142-148).

Hofmeister, Roman (1990). Handbuch der Redekunst. Rhetorik. (2 Bände). Augsburg: Weltbild Bücherdienst.

Hundt, Markus (1998). Neuere institutionelle und wissenschaftliche Wirtschaftsfachsprachen. In Hoffmann, Lothar / Kalverkämper, Hartwig / Wiegand, Herbert Ernst [Hrsg.] (1998). Fachsprachen / Languages for Special Purposes. - Ein internationales Handbuch zur Fachsprachenforschung und Terminologiewissenschaft / An International Handbook of Special-Language and Terminology Research. Band 1, Art. 143. Berlin - New York: de Gruyter. (= HSK - Handbücher zur Sprach- und Kommunikationswissenschaft. 14, 1). 1296-1304.

Kalmbach, Gabriele (1990). Kulturschock Frankreich. Bielefeld: Rump.

Kalverkämper, Hartwig (1983). Antike Rhetorik und Textlinguistik. Die Wissenschaft vom Text in altehrwürdiger Modernität. In Faust, Manfred / Harweg, Roland / Lehfeldt, Werner / Wienold, Götz [Hrsg.] (1983). Allgemeine Sprachwissenschaft, Sprachtypologie und Textlinguistik. Festschrift für Peter Hartmann. Tübingen: Narr. (= Tübinger Beiträge zur Linguistik. 215). 349-372.

Kalverkämper, Hartwig (1990). Körpersprache in der europäischen Literatur. In Hagener Universitätsreden 14 (Dies Academicus 1989), 51-91. 
Kalverkämper, Hartwig (1991). Literatur und Körpersprache. In Poetica. Zeitschrift für Sprach- und Literaturwissenschaft 23, 328-373.

Kalverkämper, Hartwig (1993). Rezension von Göttert (1991), Kopperschmidt [Hrsg.] (1990-1991), Loebbert [Hrsg.] (1991). In Rhetorik. Ein internationales Jahrbuch 12, 169-172.

Kalverkämper, Hartwig (1994). Die Rhetorik des Körpers: Nonverbale Kommunikation in Schlaglichtern. In Rhetorik. Ein internationales Jahrbuch 13: Körper und Sprache. 131-169.

Kalverkämper, Hartwig (1995). Kultureme erkennen, lehren und lernen - Eine kontrastive und interdisziplinäre Herausforderung an die Forschung und Vermittlungspraxis. In Fremdsprachen Lehren und Lernen. Zur Theorie und Praxis des Sprachunterrichts an Hochschule 24: Kontrastivität und kontrastives Lernen, 138-181.

Kalverkämper, Hartwig (1996). Im Zentrum der Interessen: Fachkommunikation als Leitgröße. In Hermes. Journal of Linguistics / Revue de Linguistique / Tidsskrift for Sprogforskning / Zeitschrift für Linguistik 16, 117-176.

Kalverkämper, Hartwig (1997). Körpersprache in älteren Kulturstufen der Romania. In Huberty, Maren / Perlick, Claudia [Hrsg.] (1997). Studia Historica Romanica. In honorem Johannes Klare. Bonn: Romanistischer Verlag. (= Abhandlungen zur Sprache und Literatur. 90). 215 - 243.

Kalverkämper, Hartwig (1998a). Interkulturalität. In Lundquist, Lita / Picht, Heribert / Qvistgaard, Jacques [Eds.] (1998). LSP. Identity and Interface. Research, Knowledge and Society. Proceedings of the $11^{\text {th }}$ European Symposium on Language for Special Purposes, Copenhagen, Aug. 1997. Copenhagen: Copenhagen Business School. 69-99.

Kalverkämper, Hartwig (1998b). Körpersprache. [Übersichtsartikel]. In Ueding, Gert [Hrsg.] (1998). Historisches Wörterbuch der Rhetorik. Tübingen: Niemeyer. 13391371.

Kalverkämper, Hartwig (1998c). Fach und Fachwissen. In Hoffmann, Lothar / Kalverkämper, Hartwig / Wiegand, Herbert Ernst [Hrsg.] (1998). Fachsprachen / Languages for Special Purposes. - Ein internationales Handbuch zur Fachsprachenforschung und Terminologiewissenschaft / An International Handbook of Special-Language and Terminology Research. Band 1, Art. 1. Berlin - New York: de Gruyter. (= HSK - Handbücher zur Sprach- und Kommunikationswissenschaft. 14, 1). 1-24.

Kalverkämper, Hartwig (1998d). Rahmenbedingungen für die Fachkommunikation. In Hoffmann, Lothar / Kalverkämper, Hartwig / Wiegand, Herbert Ernst [Hrsg.] (1998). Fachsprachen / Languages for Special Purposes. - Ein internationales Handbuch zur Fachsprachenforschung und Terminologiewissenschaft / An International Handbook of Special-Language and Terminology Research. Band 1, Art. 2. Berlin - New York: de Gruyter. (= HSK - Handbücher zur Sprach- und Kommunikationswissenschaft. 14, 1). 24-47. 
Kalverkämper, Hartwig (1999a). Vorläufer der Textlinguistik: die Rhetorik. In Antos, Gerd / Brinker, Klaus / Heinemann, Wolfgang / Sager, Sven F. [Hrsg.] (1999). Textund Gesprächslinguistik / Linguistics of Text qand Conversation. - Ein internationales Handbuch zeitgenössischer Forschung / An International Handbook of Contemporary Research. Art. Nr. 4. Berlin - New York: de Gruyter. (= HSK Handbücher zur Sprach- und Kommunikationswissenschaft).

Kalverkämper, Hartwig (1999b). Translationswissenschaft als integrative Disziplin. In Gerzymisch-Arbogast, Heidrun / Gile, Daniel / House, Juliane / Rothkegel, Annely [Hrsg.] (1998). Wege der Übersetzungs- und Dolmetschforschung. Tübingen: Narr. (= Jahrbuch Übersetzen und Dolmetschen der Deutschen Gesellschaft für Übersetzungs- und Dolmetschwissenschaft. 1). 55-76.

Kapp, Volker (1990). Die Lehre von der actio als Schlüssel zum Verständnis der Kultur der frühen Neuzeit. In Kapp [Hrsg.] (1990: 40-64).

Kapp, Volker [Hrsg.] (1990). Die Sprache der Zeichen und Bilder. Rhetorik und nonverbale Kommunikation in der frühen Neuzeit. Marburg: Hitzeroth. (= Ars rhetorica. 12).

von Keller, Eugen (1982). Management in fremden Kulturen: Ziele, Ergebnisse und methodische Probleme der kulturvergleichenden Managementforschung. Berlin: Springer.

Kopperschmidt, Josef (1973). Allgemeine Rhetorik. Einführung in die Theorie der Persuasiven Kommunikation. Stuttgart - Berlin - Köln - Mainz: Kohlhammer. (= Sprache und Literatur. 79).

Kopperschmidt, Josef (1990). Rhetorik nach dem Ende der Rhetorik. In Kopperschmidt [Hrsg.] (1990-1991), I: 1-31.

Kopperschmidt, Josef (1991). Das Ende der Verleumdung. Einleitende Anmerkungen zur Wirkungsgeschichte der Rhetorik. In Kopperschmidt [Hrsg.] (1990-1991), II: 133.

Kopperschmidt, Josef [Hrsg.] (1990-1991). Rhetorik. 2 Bände: I: Rhetorik als Texttheorie (1990). II: Wirkungsgeschichte der Rhetorik. 1991. Darmstadt: Wissenschaftliche Buchgesellschaft.

Krause, Wolfgang / Bayard, Ann-Christin (1991). Geschäftskontakte. Videosprachkurs für Wirtschaftsdeutsch. Begleit- und Arbeitsbuch. Berlin - München - Wien - Zürich - New York: Langenscheidt.

Lausberg, Heinrich (1960). Handbuch der literarischen Rhetorik. Eine Grundlegung der Literaturwissenschaft. München: Hueber. - (21989).

Lausberg, Heinrich ( $\left.{ }^{10} 1990\right)$. Elemente der literarischen Rhetorik. Eine Einführung für Studierende der klassischen, romanischen, englischen und deutschen Philologie. Ismaning: Hueber. - $\left({ }^{1} 1963\right)$.

Loebbert, Michael F. [Hrsg.] (1991). Rhetorik. Stuttgart: Reclam. (= Arbeitstexte für den Unterricht. 15021).

Messing, Ewald E. J. [Hrsg.] (1932). Zur Wirtschaftslinguistik. Rotterdam. 
Molcho, Samy (1983). Körpersprache. München: Mosaik.

Molcho, Samy (1997). Körpersprache im Beruf. München: Goldmann.

Morris, Desmond (1986). Körpersignale. Bodywatching. München: Heyne.

Müller, Bernd-Dietrich [Hrsg.] (21993). Interkulturelle Wirtschaftskommunikation. 2 überarb. u. erw. Aufl. München: Iudicium.

Müller, Cornelia (1994). Cómo se llama ...? Kommunikative Funktionen des Gestikulierens in Wortsuchen. In König, Peter-Paul / Wiegers, Helmut [Hrsg.] (1994): Satz - Text-Diskurs. Akten des 27. Linguistischen Kolloquiums, Münster 1992. Tübingen: Niemeyer. 71-80.

Müller, Cornelia (1998a). Redebegleitende Gesten. Kulturgeschichte - Theorie Sprachvergleich. Berlin: Berlin Verlag. (= Körper - Zeichen - Kultur / Body - Sign Culture. 1).

Müller, Cornelia (1998b). Beredte Hände. Theorie und Sprachvergleich redebegleitender Gesten. In Schmauser / Noll [Hrsg.] (1998: 21-44).

Oeser, Erhard / Picht, Heribert (1998). Terminologieforschung in Europa: ein historischer Überblick. In Hoffmann, Lothar / Kalverkämper, Hartwig / Wiegand, Herbert Ernst [Hrsg.] (1998). Fachsprachen / Languages for Special Purposes. - Ein internationales Handbuch zur Fachsprachenforschung und Terminologiewissenschaft I An International Handbook of Special-Language and Terminology Research. Band 1, Art. 31. Berlin - New York: de Gruyter. (= HSK - Handbücher zur Sprach- und Kommunikationswissenschaft. 14, 1). 341-347.

Oksaar, Els (1988). Kulturemtheorie. Ein Beitrag zur Sprachverwendungsforschung. Göttingen: Vandenhoeck \& Ruprecht. (= Beiträge aus den Sitzungen der Joachim Jungius-Gesellschaft der Wissenschaften, Hamburg. 6, 1988, Heft 3).

Padilla Gálvez, Jesús [Ed.] (1998). El lenguaje económico. Lengua de especialidad, Comunicación, Programas / Language of Economics. Language for Special Purposes, Communication, Programme / Wirtschaftssprache. Fachsprachen, Koтmunikation, Programme. Linz: Trauner. (= Simposium internacional).

Pfaff, Walter / Keil, Erika / Schläpfer, Beat [Hrsg.] (1996). Der sprechende Körper. Texte zur Theateranthropologie. Zürich: Museum für Gestaltung - Berlin: Alexander Verlag.

Picht, Heribert (1998). Wirtschaftslinguistik: ein historischer Überblick. In Hoffmann, Lothar / Kalverkämper, Hartwig / Wiegand, Herbert Ernst [Hrsg.] (1998). Fachsprachen / Languages for Special Purposes. - Ein internationales Handbuch zur Fachsprachenforschung und Terminologiewissenschaft / An International Handbook of Special-Language and Terminology Research. Band 1, Art. 30. Berlin New York: de Gruyter. (= HSK - Handbücher zur Sprach- und Kommunikationswissenschaft. 14, 1). 336-341.

Quintilianus, Marcus Fabius (1972). Institutionis oratoriae libri XII / Ausbildung des Redners. Zwölf Bücher. Hrsg. u. übers. v. Helmut Rahn. 1. Teil: Buch I-VI (1972); 2. Teil: Buch VII-XII (1975). Darmstadt: Wissenschaftliche Buchgesellschaft. (= Texte zur Forschung. 2, 3). - ( ${ }^{2}$ durchges. $\left.1988 ;{ }^{3} 1995\right)$. 


\section{2}

Rowland, Diana (1994). Japan-Knigge für Manager. Übers. v. Friedrich Mader. Frankfurt/M. - New York: Campus.

Rückle, Horst (1991). Körpersprache verstehen und deuten. Niedernhausen/Ts.: Falken.

Rückle, Horst $\left({ }^{8} 1991\right)$. Körpersprache für Manager. Signale des Körpers erkennen und erfolgreich umsetzen. Landsberg am Lech: Verlag Moderne Industrie. - ( $\left.{ }^{1} 1981\right)$.

Schenda, Rudolf (1998). Gut bei Leibe. Hundert wahre Geschichten vom menschlichen Körper. München: Beck.

Schmauser, Caroline (1998). Körperbewegungen und ihre Bedeutungen im Don Quijote von Cervantes. In Schmauser / Noll [Hrsg.] (1998: 59-76).

Schmauser, Caroline / Noll, Thomas [Hrsg.] (1998). Körperbewegungen und ihre Bedeutungen. Berlin: Berlin Verlag. (= Körper - Zeichen - Kultur / Body - Sign Culture. 2).

Schröder, Hartmut (1993). Interkulturelle Fachkommunikationsforschung. Aspekte kulturkontrastiver Untersuchungen schriftlicher Wirtschaftskommunikation. In Bungarten, Theo [Hrsg.] (1993). Fachsprachentheorie I: Fachsprachliche Terminologie - Begriffs- und Sachsysteme - Methodologie. Tostedt: Attikon. 517-550.

Schwertfeger, Bärbel / Lewandowski, Norbert (1990). Die Körpersprache der Bosse. Düsseldorf - Wien - New York: Econ.

Spillner, Bernd (1974). Linguistik und Literaturwissenschaft, Stilforschung, Rhetorik, Textlinguistik. Stuttgart - Berlin - Köln - Mainz: Kohlhammer.

Ueding, Gert (1985). Rhetorik des Schreibens. Eine Einführung. Königstein/Ts.: Athenäum. (= Athenäum-Taschenbücher. 2181).

Ueding, Gert / Steinbrink, Bernd (21986). Grundriß der Rhetorik. Geschichte-Technik - Methode. Stuttgart: Metzler. - (3überarb. u. erw. 1994).

Wallbott, Harald G. (1998). Ausdruck von Emotionen in Körperbewegungen und Körperhaltungen. In Schmauser / Noll [Hrsg.] (1998: 121-135).

Weinrich, Harald (1976): Sprache in Texten. Stuttgart.

Weinrich, Harald (1988). Über Sprache, Leib und Gedächtnis. In Gumbrecht / Pfeiffer [Hrsg.] (1988: 80-93).

Weinrich, Harald (1993). Textgrammatik der deutschen Sprache. Unter Mitarbeit von Maria Thurmair / Eva Breindl / Eva-Maria Willkop. Mannheim - Leipzig - Wien Zürich: Duden-Verlag.

Weinrich, Lotte (1992). Verbale und nonverbale Strategien in Fernsehgesprächen. Eine explorative Studie. Tübingen: Niemeyer. (= Medien in Forschung + Unterricht. Serie A. Band 36).

WirtschaftsDeutsch International. Zeitschrift für sprachliche und interkulturelle Wirtschaftskommunikation. Leipzig: Popp (Jahrgang 1, Heft 1, 1998 ff.). 